\title{
Phonetic effects of morphology and context: Modeling the duration of word-final $S$ in English with naïve discriminative learning ${ }^{1}$
}

\author{
FABIAN TOMASCHEK
}

Universität Tübingen

\author{
INGO PLAG \\ Heinrich-Heine-Universität Düsseldorf \\ MIRJAM ERNESTUS \\ Radboud University Nijmegen \\ R. HARALD BAAYEN \\ Universität Tübingen
}

(Received 20 April 2018; revised 14 May 2019)

\begin{abstract}
Recent research on the acoustic realization of affixes has revealed differences between phonologically homophonous affixes, e.g. the different kinds of final [s] and [z] in English (Plag, Homann \& Kunter 2017, Zimmermann 2016a). Such results are unexpected and unaccounted for in widely accepted post-Bloomfieldian item-and-arrangement models (Hockett 1954), which separate lexical and post-lexical phonology, and in models which interpret phonetic effects as consequences of different prosodic structure. This paper demonstrates that the differences in duration of English final $\mathrm{S}$ as a function of the morphological function it expresses (non-morphemic, plural, third person singular, genitive, genitive plural, cliticized has, and cliticized $i s$ ) can be approximated by considering the support for these morphological functions from the words' sublexical and collocational properties. We estimated this support using naïve discriminative learning and replicated previous results for English vowels (Tucker, Sims \& Baayen 2019), indicating that
\end{abstract}

[1] We are thankful to three anonymous reviewers for their very helpful comments on previous versions of this paper. The paper also profited from the feedback of the members of the audiences at UC Berkeley 2017, International Symposium of Morphology Lille 2017, 40. DGfSJahrestagung Stuttgart 2018, Mental Lexicon Conference Edmonton 2018. This study is part of an ongoing collaboration within the DFG Research Unit FOR2373 'Spoken Morphology'. We are very grateful to the Deutsche Forschungsgemeinschaft for funding this research (Grants: BA 3080/3-1 'The articulation of morphologically complex words' to Harald Baayen; PL151/8-1 'Morpho-phonetic Variation in English' to Ingo Plag and Mirjam Ernestus; PL151/7-1 'FOR 2737 Spoken Morphology: Central Project' to Ingo Plag). 
segment duration is lengthened under higher functional certainty but shortened under functional uncertainty. We discuss the implications of these results, obtained with a wide learning network that eschews representations for morphemes and exponents, for models in theoretical morphology as well as for models of lexical processing.

KEYWORDS: acoustic duration, discriminative learning, lexical processing, morphology, phonetics

\section{INTRODUCTION}

Many studies have shown that the phonetic realization of words may depend on the morphological structure of the word. For example, Kemps et al. (2005a, b) and Blazej \& Cohen-Goldberg (2015) showed that free and bound variants of a stem differ acoustically and that listeners make use of such phonetic cues in speech perception. Paradigmatic probability has been demonstrated to influence the duration of linking elements in Dutch compounds (Kuperman et al. 2007) and the dispersion of vowels in Russian verbal suffixes (Cohen 2015). Syntagmatic probability influences the duration of the regular plural suffix in English (Rose 2017), and the duration of third person singular $-s$ in English is subject to both syntagmatic and paradigmatic probabilities (Cohen 2014a). Some studies have found that the phonetic properties of segments vary according to the strength of the morphological boundary they are adjacent to (e.g. Smith, Baker \& Hawkins 2012, Lee-Kim, Davidson \& Hwang 2013), and others provided evidence that the duration of affixes is dependent on the segmentability of the affix (e.g. Hay 2007, Plag et al. 2017).

Several studies have investigated phonologically homophonous affixes with quite unexpected results. Ben Hedia \& Plag (2017) found that the nasal consonant of the locative prefix im- (as in import, implant) is shorter than the one in words with negative in- (impossible, impotent). Plag et al. (2017) investigated multi-functional word-final [s] and [z] in conversational North American English, using a rather small sample from the Buckeye corpus with manual phonetic annotation (Pitt et al. 2007). Their data showed robust differences in the acoustic durations of seven kinds of final [s] and [z] (non-morphemic, plural, third person singular, genitive, genitive plural, cliticized has, and cliticized $i s$ ). Basically, the same patterns of durational differences hold for New Zealand English, as shown in a study based on a very large sample with automatic phonetic annotation from the QuakeBox corpus (Zimmermann 2016a). Seyfarth et al. (2018) also found differences in stem and suffix durations in English S-inflected words (e.g. frees, laps) compared to their simplex phonologically homophonous counterparts (e.g. freeze, lapse). All of these recent findings challenge traditional models of phonology-morphology interaction and of speech production which postulate that phonetic processing does not have access to morphological information (e.g. Chomsky \& Halle 1968, Kiparsky 1982, Levelt \& Wheeldon 1994, Levelt, Roelofs \& Meyer 1999). 
In this paper, we concentrate on word-final $[\mathrm{s}]$ and $[\mathrm{z}]$ (from now on $\mathrm{S}$ ) in English and address the question of how the differences between the different types of word-final $\mathrm{S}$ observed by Plag and colleagues and by Zimmermann can be explained (Zimmermann 2016a, Plag et al. 2017). Plag et al. (2017) discuss a number of possible explanations for their findings, none of which were found to be satisfactory.

It is well known from many studies that various (conditional) probabilities predict aspects of the speech signal (e.g. Bybee 2001, Jurafsky et al. 2001a, b, Bell et al. 2003, Pluymaekers, Ernestus \& Baayen 2005b, a, Bell et al. 2009, Torreira \& Ernestus 2009). In the case of final S, however, the usual measures of experience (lexical frequency, transitional phoneme probability, neighborhood density, bigram frequency, etc.) do not appear to account for the differences in S duration. As reported by Plag et al. (2017), inclusion of these measures in regression models does not render superfluous the factor distinguishing between the different functions realized with S.

In this paper, we follow up on a study by Tucker et al. (2019) which made use of naïve discriminative learning to predict the acoustic duration of the stem vowels of English regular and irregular verbs. Naïve discriminative learning uses wide learning networks to study the consequences of error-driven learning for language and language processing. These networks make it possible to study in detail the 'discriminative capability' of linguistic cues, i.e. how well morphological functions such as those realized with the English S exponent are discriminated by sublexical and collocational features.

The study of Tucker et al. (2019) calls attention to two opposing forces shaping the duration of verbs' stem vowels. When sublexical and collocational features support strongly and directly a verb's tense, this verb's vowel has a longer duration for the majority of data points. Conversely, when features support different semantic functions, vowel duration is reduced. In what follows, we investigate whether the findings of Tucker et al. generalize and also contribute to clarifying the variation in the duration of $\mathrm{S}$ as a function of the morphological function it realizes.

To do so, we proceed as follows. We begin with a more detailed introduction to the duration of S. We then proceed with a corpus study of S in the full Buckeye, extending and replicating the results of the original Plag et al. (2017) study. This is followed by an introduction to naïve discriminative learning (NDL) and specific NDL measures such as activation or activation diversity that we use to predict the duration of S. Application of these measures to the Buckeye data shows that indeed these measures provide improved prediction accuracy. We conclude with a discussion of the theoretical implications of this result, which is non-trivial as it is obtained with a computational model that eschews form units such as morphemes or exponents and instead estimates discriminative capability directly from lowlevel form features. 


\section{FINAL S IN ENGLISH}

Homophony has attracted considerable attention in recent years as a testbed for theories of the mental lexicon. Research on lexemes has shown that homophonous lexemes show striking phonetic differences (e.g. Gahl 2008, Drager 2011). Gahl (2008) investigated the acoustic realization of 223 supposedly homophonous word pairs such as time and thyme and found that, quite consistently, the more frequent members of the pairs, e.g. time, are significantly shorter than the corresponding less frequent ones, e.g. thyme (see Lohmann (2018b) for a replication and Lohmann (2018a) for a replication with homophonous nounverb pairs). This can be taken as evidence that two homophonous lexemes cannot be represented exclusively by one identical phonological form with information on their combined frequency but that the individual frequencies must be stored with the respective lemmas and have an effect on their articulation. Similarly, Drager (2011) found that the different functions of like go together with different acoustic properties. Whether like is used as an adverbial, as a verb, as a discourse particle, or as a quotative lexeme has an effect on several phonetic parameters, i.e. the ratio of the duration of $/ 1 /$ to vowel duration, on the pitch level, and on the degree of monophthongization of the vowel /aI/. These fine differences indicate that homophony of two or more lemmas at the phonetic level may not exist (see Podlubny, Geeraert \& Tucker 2015 for a replication in Canadian English).

Similar findings seem to hold for stems or affixes. Thus, Smith et al. (2012) found acoustic differences (in durational and amplitude measurements) between morphemic and non-morphemic initial mis- and dis- (as in, e.g. distasteful vs. distinctive). Kemps et al. (2005b) provided evidence that free and bound variants of a base (e.g. help without a suffix as against help in helper) differ acoustically, even if no morpho-phonological alternations apply, and that Dutch and English listeners make use of such phonetic cues in speech perception (see also Kemps et al. 2005a).

The homophony of morphemic sounds and their non-morphemic counterparts in English have also been investigated for some time. In particular, there are some previous studies available that have investigated the phenomenon that is the topic of the present paper: word-final S in English. ${ }^{2}$

One early study of S is that of Walsh \& Parker (1983). Walsh \& Parker (1983) tested plural /s/ against non-morphemic /s/ in a reading experiment and found that the plural $\mathrm{S}$ had longer mean durations than non-morphemic $\mathrm{S}$. The authors did not use a statistical test, nor did they use a multivariate statistical analysis with

[2] There have also been some studies of English word-final /t/ and /d/ (Losiewicz 1992, Zimmermann 2016b, Seyfarth et al. 2018). These studies have mostly failed to find any significant difference between the duration of past-tense $/ \mathrm{t} /$ and $/ \mathrm{d} /$ and non-morphemic $/ \mathrm{t} / \mathrm{and} / \mathrm{d} /$. Losiewicz claims to have found a significant difference, but a reanalysis of her data using a mixed-effect regression and pertinent covariates shows a null effect for past tense versus nonmorphemic /t/ and /d/. Zimmermann (2016b) finds a significant contrast between the duration of the clitics of would and had and one between would and non-morphemic /d/. 
pertinent lexical and phonetic covariates. A reanalysis of the dataset using mixedeffect regression and additional covariates carried out by the second author of the present study showed that the data do not bear out the effects that the authors claimed they did (Plag 2014).

In a more recent study, Song et al. (2013) found a significant difference between plural, which is $7 \mathrm{~ms}$ longer, and non-morphemic /z/ in utterance-final position but not in non-final position. Song et al.'s study is based on conversational speech, but their dataset is very restricted (only monosyllables and nine different word types). Furthermore, the set of covariates taken into account was small and potential variability in voicing was not included in the analysis. Furthermore, Song et al.'s data is child-directed speech, which has been shown to differ from inter-adult speech in various ways (see, e.g. Foulkes, Docherty \& Watt 2005 for an overview and discussion).

Addressing some of the problems of earlier work, Plag and colleagues investigated final $\mathrm{S}$ in a sample of 644 English words (segmented manually) with conversational speech data from the Buckeye speech corpus (Plag et al. 2017). They measured the absolute duration of $S$ in non-morphemic /s/ and /z/ and of six different English /s/ and /z/ morphemes (plural, genitive, genitive plural, and third person singular, as well as cliticized forms of has and $i s$ ), as well as their relative duration (i.e. the ratio of $\mathrm{S}$ duration and whole word duration). As the present study is primarily geared toward explaining the findings of that study, we will look at them in more detail.

The authors used regression models that predicted the absolute or relative duration of $\mathrm{S}$ based on the type of morpheme and a number of covariates that are known to influence segmental durations, such as local speech rate, stem duration, base frequency, number of previous mentions, bigram frequency, neighborhood density, the number of consonants in the rhyme before the final $\mathrm{S}$, the voicing of $\mathrm{S}$, the following phonetic context, and the position of the word in the utterance.

In general, there are fewer significant contrasts between the different morphological categories for voiced than for unvoiced realizations of $\mathrm{S}$, which is partly due to the lack of statistical power (the voiced subset is quite small) and partly due to the fact that the voiced instances are usually shorter, which makes it more difficult to find significant differences. Still, there are four significant contrasts for voiced realizations: third person singular $[\mathrm{z}]$ is shorter than plural, genitive, and genitive-plural [z] and plural [z] is significantly longer than the voiced is clitic.

For unvoiced $\mathrm{S}$, there are 10 significant contrasts (out of 21 possible pairwise contrasts). In this subset, non-morphemic $S$ is longer than all types of morphemic S. The two suffixes (plural and third person singular) are shorter than non-morphemic $\mathrm{S}$ but longer than the two clitics of has and is. The clitics are significantly shorter than the third person singular $\mathrm{S}$ and the plural $\mathrm{S}$. 
With relative durations, there are even more significant contrasts ( 8 for $/ \mathrm{z} /$ and 12 for $/ \mathrm{s} /$ ), patterning similarly to the absolute duration differences, i.e. contrasts between plural and the rest for voiced realizations and among non-morphemic, suffixal, and clitic $S$ for unvoiced realizations.

In another study of conversational speech, Zimmermann (2016a) found phonetic effects in New Zealand English that are very similar to those of Plag et al. (2017). The same durational contrasts were found, plus a few more. Zimmermann's results were based on a very large sample of over 6900 automatically segmented words from the QuakeBox corpus (Walsh et al. 2013).

In a recent experimental study, Seyfarth et al. (2018) investigated homophone pairs and found suffixal [s] and [z] to be longer than non-morphemic [s] and [z] in otherwise homophonous monosyllabic word pairs. This contradicts the findings from the conversational speech data, and it is unclear how this difference arises. Plag and colleagues used natural speech data and Seyfarth and colleagues made up dialogues in an experiment. Plag and colleagues sampled words across the board and Seyfarth and colleagues investigated differences between actual homophones. While using homophones may control for the influence of contextual phonetic parameters, it may also introduce unclear variation since the processing of homophones may differ from that of non-homophones. Furthermore, Seyfarth and colleagues did not properly distinguish between different kinds of morphemic $S$, with unclear consequences for the results. Sixteen out of the 26 words with morphemic S involved plurals and 10 involved 3rd person singular S. Twenty out of the 26 stimuli pairs had final [z] and not [s]. This means that the majority of the morphemic stimuli were voiced plurals. Interestingly, both Plag et al. (2017) and Zimmermann (2016b) find that voiced plural $S$ is indeed significantly longer than non-morphemic voiced S, which is actually in line with Seyfarth et al.'s results for this constellation of voicing and morphemic status.

In summary, both Plag et al. (2017) and Zimmermann (2016b) have found rather complex patterns of durational differences between different types of $\mathrm{S}$ in conversational speech. The findings are robust across corpora and across varieties. In their theoretical discussion, the authors show that no extant theory can account for these facts. Strictly feed-forward models of speech production (such as Levelt et al. 1999) or theoretical models of morphology-phonology interaction (e.g. Kiparsky 1982, Bermúdez-Otero 2018) rely on the distinction of lexical versus post-lexical phonology and phonetics, and they exclude the possibility that the morphemic status of a sound influences its phonetic realization since this information is not available at the articulation stage.

Prosodic phonology (e.g. Nespor \& Vogel 2007) is a theory in which prosodic constituency can lead to phonetic effects (see, e.g. Keating 2006, Bergmann 2015). While it can account for some of the differences between homophonous morphemes with different morphological functions (e.g. durational differences between the free and bound variants of a stem (Kemps et al. 2005b), it cannot 
explain all of them. Importantly, this approach is unable to explain the patterning of the contrasts we find for final $\mathrm{S}$ in English. ${ }^{3}$

It is presently unclear how the observed differences in the duration of word-final $\mathrm{S}$ can be accounted for. In this paper, we investigate whether these differences can be understood as a consequence of error-driven learning of words' segmental and collocational properties. In order to do so, we first extend the original study of Plag et al. (2017), which was based on a small and manually segmented sample from the Buckeye corpus, to the full Buckeye corpus (Pitt et al. 2007). After replicating the differences in $\mathrm{S}$ duration, we introduce a naïve discriminative learning and train a wide learning network on the Buckeye corpus. Three measures derived from the resulting network are found to be predictive for $\mathrm{S}$ duration and improve on a statistical model that includes a factor for the different functions that can be realized with $\mathrm{S}$. We conclude with a discussion of the implications of our modeling results for theoretical morphology and models of lexical processing.

\section{Replication of Plag et al. ${ }^{4}$}

Plag et al. (2017) based their investigation on a sample from the Buckeye corpus (Pitt et al. 2007). The Buckeye corpus is a corpus of conversational speech containing the recordings from 40 speakers in Columbus, Ohio, speaking freely with an interviewer (stratified on age and gender: 20 female, 20 male, 20 old, and 20 young). The style of speech is unmonitored casual speech. The corpus provides orthographic transcriptions as well as wide and narrow time-aligned phonetic transcriptions at the word and segment level. We redid the analysis of Plag et al. (2017) on the full Buckeye corpus, using the segmentations that this corpus makes available.

We extracted all words which end in [s] or [z], resulting in a total of $28928 \mathrm{~S}$ segments. Table 1 shows the number of tokens depending on morphological function and voicing investigated in the replication. Extraction was based on the narrow phonetic transcription. Information about the grammatical status of a given $\mathrm{S}$ instance was coded automatically on the basis of the part-of-speech information of the target word and the following word as provided in the corpus.

For this substantially larger dataset, a Box-Cox analysis indicated that a logarithmic transformation of $\mathrm{S}$ duration would make the data more normaldistribution-like. The predictor of interest is the morphological function that the $S$ exponent realizes (EXPONENTFOR), with NON-MORPHEMIC, 3RDSG, GEN, HAS/IS, PL-GEN, PLURAL, and NON-MORPHEMIC as reference levels. Unlike

[3] The existing prosodic phonological literature on final S in English (e.g. Goad 1998, Goad, White \& Steele 2003) posits three different kinds of prosodic configurations in which final S may occur. However, these configurations, and the predictions that may follow from them, do not match the patterns of acoustic duration differences found in the data. See Plag et al. (2017: 210) for a more detailed discussion.

[4] All analyses can be found in the Supplementary Material downloadable from https://osf.io/ anms $7 /$. 


\begin{tabular}{lrr}
\hline & Voiced & Unvoiced \\
\hline s & 1470 & 10141 \\
3rdSg & 832 & 2846 \\
GEN & 42 & 180 \\
Has/is & 622 & 5133 \\
PL-GEN & 0 & 12 \\
Plural & 1367 & 6095 \\
\hline
\end{tabular}

Table 1

Number of $\mathrm{S}$ tokens in each morphological function split by voicing for the replication study $(\mathrm{s}=$ non-morphemic final $\mathrm{S}, 3 \mathrm{rdSg}=3 \mathrm{rd}$ person singular, $\mathrm{GEN}=$ genitive, PL-GEN = plural genitive).

Plag et al. (2017), we collapsed the has and is clitics into one class, as it is not possible to differentiate between the two by means of automatic pre-processing.

Following Plag et al. (2017), we included several predictors as controls. A factor VoICING (with levels voiced and unvoiced) was implemented indicating whenever a periodic pitch pulse was present in more than $75 \%$ of the duration of the segment. A factor MANNERFOLLOWING coded for the manner of articulation of the segment following S (levels ABSENT, APPROXIMANT, FRICATIVE, NASAL, PLOSIVE, and VOWEL). Random intercepts for SPEAKER and WORD were also included. A factor CLUSTER with levels 1, 2, and 3 was included to control for the number of consonants in the coda, where 1 equals a vowel-S sequence. Two covariates were included, the local speech rate and the duration of the base word. Speaking rate was calculated by dividing the number of syllables in a phrase by the duration of that phrase. As in the Plag et al. (2017) study, base word duration was strongly correlated with word frequency (Spearman's rank correlation $r-0.69$ ), and to avoid collinearity in the tested data, frequency was not included as a predictor (see Tomaschek, Hendrix \& Baayen 2018 b for effects of collinearity in regression analyses). We used linear mixed-effect regression as implemented in the lme4 package (version: 1.1-12 Bates et al. 2015) using treatment coding for all factors.

Table 2 presents the estimates of the coefficients of the model and the corresponding standard errors and $t$-values. In order to establish which morphological functions differed in mean durations, we tested all pair-wise contrasts between the different types of $S$ using the difflsmeans function from the lmerTest package (Kuznetsova, Brockhoff \& Bojesen Christensen 2014).

Compared to monomorphemic words ending with $\mathrm{S}, \mathrm{S}$ duration was shorter when S realized PLURAL, 3RDSG, GEN, and HAS/IS. Plag et al. (2017) observed a difference as well for genitive plurals, but for the full Buckeye, this contrast was not supported. Furthermore, as in the study of Plag et al. (2017), the S was articulated with shorter duration when realizing HAS or IS compared to when it realizes plurals or the third person singular. Plag et al. (2017) observed an 


\begin{tabular}{lrrrr}
\hline & Estimate & Std. error & df & $t$-Value \\
\hline Intercept & -1.52 & 0.02 & 148.39 & -69.93 \\
ExponentFor $=$ 3rdSg & -0.10 & 0.02 & 1372.72 & -5.65 \\
ExponentFor $=$ GEN & -0.15 & 0.03 & 5647.45 & -5.46 \\
ExponentFor $=$ has/is & -0.15 & 0.02 & 1416.32 & -7.33 \\
ExponentFor $=$ PL-GEN & -0.12 & 0.11 & 5778.72 & -1.08 \\
ExponentFor $=$ plural & -0.10 & 0.01 & 1380.73 & -8.98 \\
Voicing $=$ unvoiced & 0.23 & 0.01 & 28924.37 & 35.66 \\
Cluster $=2$ & -0.19 & 0.01 & 5778.52 & -26.03 \\
Cluster $=3$ & -0.29 & 0.01 & 6103.94 & -19.73 \\
MannerFollowing $=$ app & -0.31 & 0.01 & 28822.04 & -37.63 \\
MannerFollowing $=$ fri & -0.52 & 0.01 & 28900.28 & -71.39 \\
MannerFollowing $=$ nas & -0.47 & 0.01 & 28872.42 & -31.94 \\
MannerFollowing $=$ plo & -0.51 & 0.01 & 28906.19 & -72.46 \\
MannerFollowing $=$ vow & -0.43 & 0.01 & 28909.55 & -62.94 \\
LocalSpeechRate & -0.08 & 0.00 & 28837.16 & -38.43 \\
BaseDuration & 0.19 & 0.01 & 16193.21 & 32.88 \\
\hline
\end{tabular}

Table 2

Coefficients and associated statistics for the mixed-effect model fit to the log-transformed duration of $\mathrm{S}$, using the full Buckeye corpus ( app = approximant, fri = fricative, nas $=$ nasal, plo $=$ plosive, vow $=$ vowel $)$.

interaction of EXPONENTFOR by VOICING, but this interaction did not replicate for the enlarged dataset. The differences between the present analysis and that of Plag et al. (2017) have two possible sources. First, Plag et al. (2017) manually inspected all data points and curated the automatic annotations and segmentations where necessary. By contrast, we followed the annotations and segmentations provided by the Buckeye corpus, which are also generally manually corrected on the basis of forced alignments. It is unclear at what level of carefulness the original manual corrections of the Buckeye corpus were performed. In addition, whereas misalignment tends to be very consistent and systematic in forced aligners, human annotators can be biased by their own expectations and create different kinds of variations in the annotation (Ernestus \& Baayen 2011). Therefore, there is no way to know which annotation can be strongly relied on, especially for phones with gradual transitions such as sonorants. Second, by considering the full corpus, the present analysis is possibly somewhat more robust against spurious small-sample effects. For instance, in the dataset of Plag et al. (2017), there were only 81 voiced $\mathrm{S}$ tokens, as opposed to 563 voiceless $\mathrm{S}$ tokens. Table 3 summarizes a comparison of the significant contrasts for unvoiced $S$ in the small sample of Plag et al. (2017) with those found in the full corpus used here. Apart from one contrast, all contrasts are significant in both datasets. 


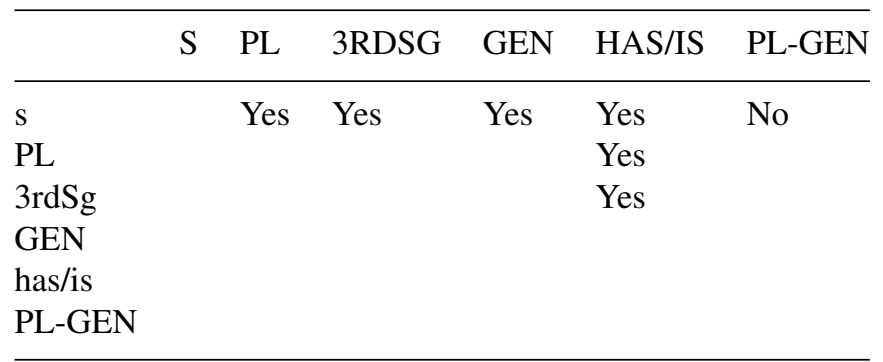

Table 3

Significant contrasts for unvoiced S in the small sample of Plag et al. (2017) and the present replication study (see Table 2). 'Yes' indicates an effect found in both studies and 'no' indicates an effect found only in the small sample, for $\alpha=0.05$ (under Tukey's HSD) ( $\mathrm{s}=$ non-morphemic final $\mathrm{S}, 3 \mathrm{rdSg}=3 \mathrm{rd}$ person singular, $\mathrm{GEN}=$ genitive, PL-GEN = plural genitive).

Two things are important to note. First, the main finding of Plag et al. (2017) is the difference in duration between unvoiced non-morphemic S (longest), clitic S, and suffix $\mathrm{S}$ (shortest). This difference is also found in the larger dataset with automatic annotation. Second, while in the Plag et al. (2017) data set there was a significant interaction between voicing and morpheme type, this interaction is no longer present in the larger dataset.

To summarize, we have replicated the main findings of Plag et al. (2017) for a much larger dataset derived from the same speech corpus. However, we still lack an explanation for the durational patterns observed. In the following sections, we will provide such an explanation, arguing that durational variation in wordfinal $S$ is chiefly influenced by how strongly the final $S$ is associated with its morphological function as a result of learning. This association strength will be derived from a naïve discriminative learning network, as explained in the next section.

\section{NAÏVE DISCRIMINATIVE LEARNING}

\subsection{General overview}

Naïve discriminative learning (NDL) is a computational modeling framework that is grounded in simple but powerful principles of discrimination learning (Ramscar \& Yarlett 2007, Ramscar et al. 2010, Baayen et al. 2011, Rescorla 1988). The general cognitive mechanisms assumed in this theory have been shown to be able to model a number of important effects observed in animal learning and human learning, e.g. the blocking effect (Kamin 1969) and the featurelabel ordering effect (Ramscar et al. 2010). NDL has recently been extended to language learning and language usage, and several studies have shown that it can successfully model different morphological phenomena and their effects onto human behavior, e.g. reaction times in experiments investigating morphological 
processing (e.g. Baayen et al. 2011, Blevins, Ackerman \& Malouf 2016; see Plag 2018: Section 2.7.7 for an introduction).

Discriminative learning theory rests on the central assumption that learning results from exposure to informative relations among events in the environment. Humans (and other organisms) use these relations, or 'associations', to build cognitive representations of their environments. Crucially, these associations (and the resulting representations) are constantly updated on the basis of new experiences. Formally speaking, the associations are built between features (henceforth cues) and classes or categories (henceforth outcomes) that co-occur in events in which the learner is predicting the outcomes from the cues. The association between cues and outcomes is computed mathematically using the so-called Rescorla-Wagner equations (Rescorla \& Wagner 1972, Wagner \& Rescorla 1972, Rescorla 1988; see Appendix A for a technical description). The equations work in such a way that the association strength or 'weight' of an association between a cue and an outcome increases every time that this cue and outcome co-occur. Importantly, this association weight decreases whenever the cue occurs without the outcome being present in a learning event. During learning, weights are continuously recalibrated. At any stage of learning, the association weight between a cue and an outcome can be conceptualized as the support which that specific cue can provide for that specific outcome given the other cues and outcomes which had been encountered during the learning history.

Let us look at an example of how our understanding of the world is constantly modulated by the matches and mismatches between our past experiences and what we actually observe. Our example is a phenomenon known as 'anti-priming' found by Marsolek (2008). He presented speakers with sequences of two pictures and asked these speakers to say the name of the second picture. The critical manipulation was implemented in the first picture, which could be either similar, to some extent, to the target picture (e.g. grand piano, followed by table), or unrelated (e.g. orange, followed by table). In contrast to typical priming findings, Marsolek observed that speakers responded more quickly for unrelated pairs compared to related pairs. This 'anti-priming' - caused by prior presentation of a related picture - follows straightforwardly from the learning rule of Rescorla \& Wagner (1972). The weights of visual features (i.e. the cues) that are shared by grand piano and table, such as having legs and a large flat surface, are strengthened for grand piano but weakened for table when the picture of the grand piano is presented. Slower response times in this case of anti-priming are a direct consequence of critical features losing strength to table compared to cases in which a visually unrelated prime, such as an orange, had been presented.

Taking a morphological example, the association of the phonological string /azz/ with a causative meaning ('make') in English would be strengthened each time a listener encounters the word modernize and weakened each time the listener hears the words size or eyes. The association strengths resulting from such experiences influence language processing in both production and comprehension. 
Technically, the mathematical engine of NDL, i.e. the Rescorla-Wagner equations, is an optimized computational implementation of an error-driven discrimination learning. This engine can be viewed as implementing 'incremental regression' (for a nearly identical algorithm from physics, see Widrow \& Hoff (1960) and for a Bayesian optimized algorithm, Kalman (1960)). NDL was first applied to large corpus data and used to study chronometric measures of lexical processing by Baayen et al. (2011). An extension of the learning algorithm is reported in Sering, Milin \& Baayen (2018b). Implementations are available both for R (Shaoul et al. 2014) and Python (Sering et al. 2018a).

Once a network has been trained, it provides different measures that represent different aspects of the association strength between cues and outcomes. These measures may subsequently be used as predictors of human responses (e.g. response times in lexical decision experiments). In the present study, we will use three NDL measures to predict the acoustic duration of $\mathrm{S}$ in regression analyses.

Other approaches to learning are available, for instance, the Bayesian model presented in Kleinschmidt \& Jaeger (2015). Where NDL comes into its own, compared to models based on probability theory, is when there are thousands or tens of thousands of different features (cues) that have to be learned to discriminate equally large numbers of classes (outcomes). Cues compete for outcomes in often unforeseeable ways reminiscent of chaotic systems, which is why it is a truly daunting challenge to capture the dynamics of such systems with probabilities defined over hand-crafted hierarchies of units (i.e. with probabilistic statistics). Errors at lower levels of the hierarchy tend to propagate to higher levels and render the performance of such models less than optimal. This is why in computational linguistics, there is a strong movement in the direction of end-toend models which bypass the engineering by hand of intermediate representations using neural networks. NDL adopts this end-to-end approach. In contrast to approaches in machine learning, however, NDL does not use any hidden layers. Rather, it makes use of the simplest possible network architecture, with just one input layer and one output layer.

NDL thus offers a simple method for assessing the consequences of discrimination learning that has hardly any free parameters (namely, only a learning rate, typically set to 0.001 , and the maximum amount of learning $\lambda$, set to 1.0). Consequently, once the representations for the input and output layers of the network have been defined and the learning rate and $\lambda$ have been set, its performance is determined completely by the corpus on which it is trained.

NDL also differs from standard applications of neural networks in machine learning in that it uses very large numbers of input and output features. We therefore refer to the NDL networks as 'wide learning' networks. The weights of these networks are updated incrementally by applying the learning rule of Rescorla and Wagner to the so-called learning events. Learning events are defined as moments in learning time at which a set of cues and a set of outcomes are evaluated jointly. Association weights between cues and outcomes are strengthened for those outcomes that were correctly predicted and weakened for all other 
outcomes. For technical details, see Milin et al. (2017b) and Sering et al. (2018b), and for a simple introductory implementation, see Plag (2018: Section 7.4.4).

This approach to simulate language learning has proved useful for, e.g. modeling child language acquisition (Ramscar et al. 2010, 2011, Ramscar, Dye \& Klein 2013a, Ramscar et al. 2013b), for disentangling linguistic maturation from cognitive decline over the lifespan (Ramscar et al. 2014, 2017), for predicting reaction times in the visual lexical decision task (Baayen et al. 2011, Milin et al. 2017b) and self-paced reading (Milin, Divjak \& Baayen 2017a), as well as for auditory comprehension (Baayen et al. 2016b, Arnold et al. 2017). The computational model developed by Arnold et al. (2017) is based on a wide learning network that has features derived automatically from the speech signal as input. This model outperformed off-the-shelf deep learning models on single-word recognition and shows hardly any degradation in performance when presented with speech in noise (see also Shafaei Bajestan \& Baayen 2018).

By adopting an end-to-end approach with wide learning, naïve discriminative learning approaches morphology, the study of words' forms and meanings, from a very different perspective than the standard post-Bloomfieldian hierarchical calculus based on phonemes, morphemes, and words. The relation between form and meaning is addressed directly, without intervening layers of representations. In what follows, we will make use of wide learning networks primarily as a convenient tool from machine learning. In Section 6, we will briefly return to the question of the implications of successful end-to-end learning for morphological theory.

\subsection{From NDL to phonetic durations}

The present study follows up on Tucker et al. (2019), who used NDL measures to predict the durations of stem vowels of regular and irregular verbs in English in the Buckeye corpus. Their NDL wide learning network had diphones as cues, and as outcomes both content lexemes (or more specifically, pointers to the meanings of content words) and morphological functions (such as plural or the clitic has). In what follows, we refer to these pointers to meanings/functions as lexomes (see Milin et al. 2017b for a detailed discussion). Tucker et al. observed that the prediction accuracy of statistical model fits to vowel duration improved substantially when classical predictors such as frequency of occurrence and neighborhood density were replaced by predictors grounded in naïve discriminative learning.

Following their lead, we implemented a network that has morphological function lexomes as outcomes, but restricted them to those that are implicated with English word-final S: CLITIC, GENITIVE PLURAL, GENITIVE SINGULAR, PLURAL NOUN, SINGULAR NOUN, THIRD PERSON VERB, VERB, VERB PARTICIPLE, PAST-TENSE VERB, and OTHER (such as adverbs). The number of morphological functions is larger than that examined in the original Plag et al. (2017) study, as we also include $S$ that is word-final in past-tense or 
past-participle forms as a result of reduction (e.g. in pass for passed). Voicing of $\mathrm{S}$ was based on the phonetic transcription provided by the Buckeye corpus.

The findings by Tucker et al. (2019) indicate that speakers have to balance opposing forces during articulation, one that seeks to lengthen parts of the signal in the presence of strong bottom-up support and one that seeks to shorten them in case of high uncertainty. To parameterize these forces, we derived three different measures from the NDL wide learning network which are used as predictors of $\mathrm{S}$ duration: the $\mathrm{S}$ lexomes' activations, their priors, and their activation diversities. Table 4 provides an example of a simple NDL network where the diphone cues for the word form 'dogs' are associated with, among others, the lexome of the morphological function PLURAL. We will discuss each measure in turn.

\begin{tabular}{|c||c|c|c|c|}
\hline & $o_{1}$ & $\{\text { plural }\}_{2}$ & $\ldots$ & $o_{n}$ \\
\hline$c_{1}$ & $w_{1,1}$ & $w_{1,2}$ & $\ldots$ & $w_{1, n}$ \\
\hline$c_{2}$ & $w_{2,1}$ & $w_{2,2}$ & $\ldots$ & $w_{2, n}$ \\
\hline $\mathrm{ld}$ & $w_{3,1}$ & $w_{3,2}$ & $\ldots$ & $w_{3, n}$ \\
\hline $\mathrm{dO}$ & $w_{4,1}$ & $w_{4,2}$ & $\ldots$ & $w_{4, n}$ \\
\hline $\mathrm{Og}$ & $w_{5,1}$ & $w_{5,2}$ & $\ldots$ & $w_{5, n}$ \\
\hline $\mathrm{gz}$ & $w_{6,1}$ & $w_{6,2}$ & $\ldots$ & $w_{6, n}$ \\
\hline $\mathrm{zb}$ & $w_{7,1}$ & $w_{7,2}$ & $\ldots$ & $w_{7, n}$ \\
\hline$\ldots$ & $\ldots$ & $\ldots$ & $\ldots$ & $\ldots$ \\
\hline$c_{k}$ & $w_{k, 1}$ & $w_{k, 2}$ & $\ldots$ & $w_{k, n}$ \\
\hline & $a_{1}$ & $a_{2}$ & $\ldots$ & $a_{n}$ \\
\hline
\end{tabular}

Table 4

The table illustrates a cue-to-outcome network with a set of cues $\mathcal{C}$ with $k$ cues $c$ and a set of lexome outcomes $\mathcal{O}$ with $n$ outcomes $o$. We illustrate the calculation of NDL measures for the lexome of the morphological function PLURAL as an outcome, located in the second column, and its associated cue set $\mathcal{C}_{\Omega}=1 \mathrm{~d} \mathrm{dO} O \mathrm{~g} \mathrm{gz} \mathrm{zb,} \mathrm{located} \mathrm{in} \mathrm{rows} \mathrm{3-7.} \mathrm{Each} \mathrm{ith}$ cue $c$ is associated with each jth outcome o by a weight $w_{i, j}$, representing their connection strength, where $i=1,2, \ldots, k$ and $j=1,2, \ldots, n$. Summed weights for $\mathcal{C}_{\Omega}$ afferent to $o_{j}$ give the $j$ th activation $a$. The cues in $c_{1}, c_{2}$ represent any kind of cues that might occur in the first and second row.

A lexome's activation represents the bottom-up support for that lexome, given the cues in the input. The activation for a given lexome is obtained simply by the summation of the weights on the connections from those cues that are instantiated in the input to that outcome (equivalent to the weights marked in red in Table 4). Hence, activation represents a measure of the cumulative evidence in the input.

A lexome's prior is a measure of an outcome's baseline activation, calculated by the sum of all absolute weights pertinent to the lexome outcome (equivalent 
to the weights in the column marked in light gray in Table 4). ${ }^{5}$ The prior can be understood as a measure of network entrenchment. It is an NDL measure that is independent of a particular input to the network; rather it captures a priori availability which results from learning and typically corresponds to frequency of occurrence.

Finally, a lexome's activation diversity is a measure of the extent to which the input makes contact with the lexicon. Activation diversity is the sum of the absolute activations pertinent from a cue set to all lexome outcomes (equivalent to the activations located in the bottom row highlighted in dark gray in Table 4). One can think of this measure as quantifying the extent to which the cues in the input perturb the state of the lexicon. If the cues were to support only the targeted outcome, leaving all other outcomes completely unaffected, then the perturbation of the lexicon would be relatively small. However, in reality, learning is seldom this crisp and clear-cut, and the states of outcomes other than the targeted ones are almost always affected as well. In summary, the more the lexicon as a whole is perturbed, the greater the uncertainty about the targeted lexomes will be.

Tucker et al. (2019) observed that vowel duration decreased with activation diversity. When uncertainty about the targeted outcome increases, acoustic durations decrease (for further examples of shortening under uncertainty, see also Kuperman et al. (2007) and Cohen (2014a)). Arnold et al. (2017) performed an auditory experiment in which subjects had to indicate whether they could identify the words presented to them. These words were randomly selected from the GECO corpus (Schweitzer \& Lewandowski 2013). Arnold et al. observed that words with low activation diversity (i.e. with short vectors that hardly penetrate lexical space) were quickly rejected, whereas words with large activation diversity (i.e. with long vectors that reach deep into lexical space) were more likely to be identified, but at the cost of longer response times.

Tucker et al. (2019) also observed that prediction accuracy decreases when instead of using the diphones in the transcription of what speakers actually said, the diphones in the dictionary forms are used. We therefore worked with diphones derived from the actual speech. However, we considered a broader range of features as cues.

Several studies that made use of discriminative learning actually worked with two networks, one network predicting lexomes from form cues, resulting in form-to-lexome networks, and the other predicting lexome outcomes from lexome cues, creating lexome-to-lexome networks (Baayen et al. 2016b, Milin et al. 2017a, b, Baayen, Milin \& Ramscar 2016a). Lexome-to-lexome networks stand in the tradition of distributional semantics (Landauer \& Dumais 1997, Lund \& Burgess 1996a, Shaoul \& Westbury 2010a, Mikolov et al. 2013). The row vectors

[5] Technically, the sum of the absolute values of a vector is the L1-norm. It is related to the L2-norm, which is the Euclidean distance. For example, the Euclidean distance for the vector $(-3,-4)$ is 5 (by Pythagoras), but the L1-norm is 7 , the distance traveled from the origin to the point $(-3,-4)$ when movement is possible only along the horizontal axis or along the vertical axis. 
of the weight matrix of lexome-to-lexome networks of NDL specify, for each lexome, the association strengths of that lexome with the full set of lexomes. These association strengths can be interpreted as gauging collocational strengths. In the present study, we do not work with a separate lexome-to-lexome network. Rather, we used a joint network that contains both lexomes and their diphones as cues and morphological functions as outcomes. ${ }^{6}$

\subsection{Cue-to-outcome structure}

Let us now turn to the actual modeling procedures that we employed and the evaluation of these models that led us to focus on inflectional lexomes as outcomes.

As a general strategy, we wanted to explore various constellations of cues and outcomes. We also considered the possibility that $\mathrm{S}$ duration might be codetermined by the lexomes in a word's immediate context. Therefore, just as in models for distributional semantics, such as those presented by Lund \& Burgess (1996b), Shaoul \& Westbury (2010b), and Mikolov et al. (2013), we placed an n-word window around a given target word and restricted cues and outcomes to features within this window. By varying the window size between zero and maximally two to the left or the right of the target word, and the specific features selected for cues and outcomes, we obtained a total of 38 NDL networks.

We created diphone cues on the basis of an entire phrase. This procedure created transition cues between words. A sequence such as 'dogs bark' gives the diphone cues dO Og gz $\mathrm{zb}$ bA Ar rk with zb as the transition cue.

Table 5 illustrates several different choices for cues and outcomes, given the phrase the small dogs bark at the cat, where dogs is the pivotal word carrying $\mathrm{S}$. Examples 1, 2, and 5 illustrate models in which lexomes are outcomes and examples 3 and 4 have diphones as outcomes. Example 1 has only diphones as cues, and this model is a standard form-to-lexome network following the approach originally taken by Baayen et al. (2011) and Tucker et al. (2019). Example 2 has lexomes as cues and outcomes; this is a standard lexome-to-lexome network (see Baayen et al. 2016a, Milin et al. 2017b for applications of such networks for modeling reaction times). Model 3 seeks to predict diphones from lexomes. Model 4

[6] Depending on the task for which the semantic vectors - in the terminology of computational linguistics, semantic vectors are called 'word embeddings' - are used, the association strength of a lexome with itself can be either left as is in the vector or set to zero (see Baayen et al. 2019 for a detailed discussion). Similarity between semantic vectors is typically evaluated with the cosine similarity measure or the Pearson correlation between vectors. Just as for formto-lexome networks, activations, priors, and activation diversity measures can be calculated for lexome-to-lexome networks. The lexomes that we use as cues or outcomes are labels for semantic vectors - in the framework of programing, specifically data structures, labels are called 'pointers' to semantic networks. Since semantic vectors define points in a high-dimensional collocational space, the lexomes of an NDL network are simply identifiers for these points. Baayen et al. (2019) updated this approach by presenting a computational model in which the lexomic pointers of NDL are replaced by semantic vectors. 


\begin{tabular}{|c|c|c|}
\hline & Cues & Outcomes \\
\hline \multirow{5}{*}{$\begin{array}{l}1 \\
2 \\
3 \\
4\end{array}$} & Te es sm m6 61 ld do Og gz zb ba ar rk ke det & DOGS DOG PLURAL \\
\hline & THE SMALL DOGS BARK AT & DOGS DOG PLURAL \\
\hline & THE SMALL DOGS BARK AT & ld dO Og gz zb \\
\hline & THE SMALL DOGS BARK AT & \\
\hline & Te es sm m6 61 ld do Og gz zb ba ar rk ke det & ld dO Og gz zb \\
\hline \multirow[t]{2}{*}{5} & THE SMALL DOGS BARK AT & \\
\hline & Te es sm m6 61 ld do Og gz zb ba ar rk ke at & DOGS DOG PLURAL \\
\hline
\end{tabular}

Table 5

Possible cue-outcome configurations for the phrase the small dogs bark at the cat using a five-word window centered on dogs. 
complements the lexome cues with diphone cues. Model 5 also combines lexomes and diphones as cues, but these are used to predict lexomic outcomes. Importantly, these lexomes include the inflectional lexomes that are realized with $\mathrm{S}$ in English. The pertinent lexome in the present example is the one for plural number (PLURAL). Note that model 5 allows us to test the hypothesis that the support for PLURAL is obtained not only from a word's diphones but also from its collocates.

Models were trained by moving a given word window across the whole of the Buckeye corpus. ${ }^{7}$ The window was moved across the corpus such that each word token was in the center of the window once. Consequently, a given $\mathrm{S}$ word will have occurred in each of the positions in the window. Each window provided a learning event at which prediction accuracy was evaluated and connection weights were recalibrated.

A wide variety of selections of cues and outcomes was investigated with the aim of obtaining insight into which combinations of cues and outcomes, under a discriminative learning regime, best predict $S$ duration. Models with lexomes as outcomes, specifically those for the morphological functions of the $S$ (CLITIC, GENITIVE PLURAL, GENITIVE SINGULAR, PLURAL NOUN, SINGULAR NOUN, THIRD PERSON VERB, VERB, VERB PARTICIPLE, PAST-TENSE VERB, and OTHER) address the possibility that it is the learnability of the inflectional lexomes that drives the acoustic duration of $\mathrm{S}$. Models that take diphones as outcomes address the hypothesis that it is the learnability of diphones (i.e. of context-sensitive phones) that is at issue.

In classical models of speech production, e.g. the WEAVER model of Levelt et al. (1999) and the model of Dell (1986), the flow of processing goes from conceptualization to articulation. Against this background, models in which lexomes are predicted instead of being predictors are unexpected. Nevertheless, there are three reasons why it makes sense to include such models in our survey.

First, for a survey, it is important to consider a wide range of possible combinations, including ones that are at first sight counter-intuitive. This is essential for allowing data to inform theory.

The second reason is technical in nature: NDL makes the simplifying assumption that each outcome can be modeled independently from all other outcomes. It is this assumption that motivates why NDL is referred to as nä̈ve discriminative learning. For discriminative learning to take place, multiple cues are required for a given outcome so that over learning time it can become clear, due to cue competition, which cues are informative and which are uninformative. Informative cues obtain larger association strengths and uninformative cues obtain association strengths close to zero. If the learnability of inflectional lexomes is what drives $\mathrm{S}$ duration, then the NDL network must include inflectional lexomes as outcomes.

[7] The corpus contains 286,982 words. The learning rate $\alpha \beta$ was set to 0.001 and $\lambda$ was set to 1.0 ; these are the default settings, and these parameters were never changed. The lexome for morphological function of words without final S was marked as 'other'. 
If we were to take these inflectional lexomes as cues and use them to predict a diphone such as $g z$ as outcome, the network would only learn the relative frequencies with which the inflectional lexomes are paired with $g \mathrm{z}$ in the corpus (cf. Ramscar et al. 2010).

Third, any production system must have some form of feedback control so that the sensory consequences of speaking can be evaluated properly. Without such feedback, which comprises sensory feedback from the articulators as well as proprioceptive feedback from hearing one's own speech, learning cannot take place (see Hickok 2014 for a detailed discussion). For the error-driven learning to be at all possible, distinct articulatory and acoustic targets must be set up before articulation, against which the feedback from the articulatory and auditory systems can be compared. In what follows, the diphone outcomes are a crude approximation of the speaker's acoustic targets, and the connections from the diphones to the lexomes are part of the speech control loop. For a computational model providing a more detailed proposal for resonance between the production and comprehension systems, see Baayen et al. (2019).

\subsection{NDL measures as predictors}

Having trained the 38 networks, we then analyzed their performance using random forests (as implemented in the party package for $\mathrm{R}$ ), focusing on the variable importance of the NDL measures derived from these networks. The optimal network that emerged from this analysis is the one with a five-word window and the structure of example 5 in Table 5. Critical lexomes, i.e. morphological functions, were predicted from all lexomes and their diphones within a fiveword window centered on the target word. Given the literature on conditional probabilities for upcoming (or preceding) information, such as the probability of the current word, given the next word (Jurafsky et al. 2000, Pluymaekers et al. 2005b, Tremblay et al. 2011, Bell et al. 2009), we included in our survey of cue and outcome structures windows of size three, with the target word in either first or second position. The corresponding networks lacked precision compared to the above network trained on learning events of five words. ${ }^{8}$ The latter network is also sensitive to co-occurrence of the target word with the preceding and upcoming word, but it is sensitive as well to co-occurrence with words further back and further ahead in time.

[8] For instance, we compared statistical models using the NDL measures derived from the model with a five-word window as predictors for $\mathrm{S}$ duration with statistical models with NDL measures derived from models using three-word windows, with the target word either at the left or at the right position. Statistical models with measures derived from the NDL networks based on three-word windows performed worse, with larger ML scores $(+23.31 /+83.16)$ than the statistical model based on the network models trained with a five-word window. We also tested the performance of a statistical model based on an NDL network trained with a five-word window, but using only the diphones but not the words. The resulting statistical model yielded a higher ML score as well $(+160.16)$. These three alternative mixed models had as many degrees of freedom as the five-word model (31), hence all these alternative models underperformed in terms of goodness of fit. 
In the light of the literature on boundary strength and its consequences for lexical processing (Seidenberg 1987, Weingarten, Weingarten \& Will 2004, Hay 2002, 2003, Hay \& Baayen 2002, Baayen et al. 2016b, 2019), we considered separately the activation and activation diversity calculated for the diphone straddling the boundary between stem and $\mathrm{S}$, and the activation and activation diversity calculated from all other remaining cues (lexomes and diphones). This resulted in a total of five NDL measures as predictors of S duration:

1. PRIORMORPH: the prior for weights from a cue set to a word's inflectional lexome.

2. ACTFROMBOUNDARYDIPHONE: the activation of an inflectional lexome by the boundary diphone.

3. ACTFROMREMAININGCUES: the activation of an inflectional lexome by all other (lexome and diphone) cues.

4. ACTDIVFROMBOUNDARYDiphone: the activation diversity calculated over the vector of activations over all inflectional lexomes of $\mathrm{S}$, given the boundary diphone as cue.

5. ACTDIVFROMREMAININGCUES: the activation diversity, again calculated over the vector of activations of all inflectional lexomes, but now using the remaining cues in the learning event.

There are nine values that PRIORMORPH can assume, one value for each of the nine inflectional lexomes that we distinguished (CLITIC, GENITIVE PLURAL, GENITIVE SINGULAR, PLURAL NOUN, SINGULAR NOUN, THIRD PERSON VERB, VERB, VERB PARTICIPLE, PAST-TENSE VERB, and OTHER). The boundary diphone will usually differ from word to word depending on the stem-final consonant and the specific realization of the S. For any specific boundary diphone, there are again nine possible values of ACTFROMBOUNDARYDIPHONE and ActDivFromBoundaryDiphone, one for each inflectional lexome. For a given target word, e.g. dogs, we consider the activation and activation diversity, given [gz] as cue, for the corresponding inflectional outcome, here NOUN PLURAL. The values of ACTFROMREMAININGCUES and ACTDIVFROMREMAININGCUES depend on the words that happen to be in the moving window, and hence their values vary from token to token. In this way, each target word was associated with five measures for its inflectional lexome.

Although the prior, activation, and activation diversity measures have been found to be useful across many studies, there is considerable uncertainty about how they might predict the duration of English S.

With respect to PRIORMORPH, the general strong correlation of NDL priors with word frequency would suggest, given the many studies reporting durational shortening for increasing frequency (see, e.g. Zipf 1929, Jurafsky et al. 2001a, Bell et al. 2003, Gahl 2008), that a greater PRIORMORPH correlates with shorter S. However, recent findings emerging from production studies using electromagnetic articulography suggest that a higher prior (or frequency of occurrence) might predict increased rather than decreased S duration: Tomaschek 
et al. (2018c) observed that, other things being equal, greater frequency enables speakers to execute articulatory gestures with more finesse, in parallel to the general finding that motor skills improve with practice. It is also possible that PRIORMORPH will not be predictive at all, as Tucker et al. (2019) did not observe an effect of the prior for stem vowel duration.

For the activation measures (ACTFROMBOUNDARYDIPHONE and ACTFROMREMAININGCUES), our expectation is that a greater activation will afford durational lengthening. Arnold et al. (2017) observed, using an auditory word identification task, that a greater activation corresponded to higher recognition scores. Since a higher signal to noise ratio is expected to give rise to improved recognition rates, the prediction follows for English $S$ that when the activation is higher, there must be more signal compared to noise, and this higher signal to noise ratio is, for a fricative such as $\mathrm{S}$, likely to be realized by lengthening. This is indeed what Tucker et al. (2019) observed for vowel duration in regular verbs: as activation increased, the duration of the stem vowel increased likewise.

Turning to the activation diversity measure, here Tucker et al. (2019) observed a strong effect, with larger activation diversity predicting shorter duration. This result fits well with the finding of Arnold et al. (2017) that in auditory word identification, words with a low activation diversity elicited fast negative responses, whereas words with higher activation diversity had higher recognition scores that came with longer decision times. In fact, the activation diversity measure can be understood as a measure of lexicality: a low lexicality is an index of noise, whereas a high lexicality indicates that the speech signal is making contact with possibly many different words. The other side of the same coin is that discriminating the target lexome in a densely populated subspace of the lexicon takes more time. For speech production, Tucker et al. (2019) argued that when lexicality is high, the system is in a state of greater uncertainty as many lexomes are co-activated with the targeted outcome. Importantly, if some part of the signal, e.g. English S, contributes to greater uncertainty, it is disadvantageous for both the listener and the speaker to extend its duration. All that extending its duration accomplishes is that uncertainty is maintained for a longer period of time. It makes more sense to reduce the duration of those parts of the signal that do not contribute to discriminating the targeted outcome from its competitors. These considerations led us to expect a negative correlation between activation diversity and $\mathrm{S}$ duration.

\section{Results}

We analyzed the log-transformed duration of $\mathrm{S}$ with a generalized additive mixed model (GAMM, Wood 2006, 2011) with random intercepts for the speaker and the word. In addition to the five measures derived from the NDL network, we controlled for the manner of the preceding and following segment by means of two factors, one for the preceding segment and one for the following segment 
(each with levels APPROXIMANT, FRICATIVE, NASAL, PLOSIVE, VOWEL, and ABSENT). We included the average speaking rate of the speaker (INDIVIDUALSPEAKINGRATE) and the local speaking rate (LOCALSPEAKINGRATE) as control covariates.

In a number of cases, the S-bearing word would be located in a phrase final position and the last diphone cue would be $s \#$ or $z \#$. These cues resulted in strong outliers in the NDL measures, which is why these words were excluded from analysis. A total of 27091 tokens was investigated with NDL measures; Table 6 shows the number of tokens depending on function and voicing.

\begin{tabular}{lrr}
\hline & Voiced & Unvoiced \\
\hline Clitic & 1469 & 3812 \\
Genitive plural & 7 & 6 \\
Genitive singular & 81 & 31 \\
Plural noun & 3841 & 2115 \\
Singular noun & 224 & 1749 \\
Third person verb & 2326 & 968 \\
Verb & 1367 & 6095 \\
Verb participle & 72 & 88 \\
Past-tense verb & 2177 & 463 \\
Other & 2947 & 4299 \\
\hline
\end{tabular}

Table 6

Number of S tokens in each morphological function split by voicing investigated with NDL measures.

The model we report here is the result of exploratory data analysis in which the initial model included all control predictors and the random effect factors but no NDL measures. We then added in NDL measures step by step, testing for nonlinearities and interactions. Model criticism of the resulting generalized additive mixed model (GAMM) revealed that the residuals deviated from normality. This was corrected for by refitting the model with a GAMM that assumes that the scaled residuals follow a $t$-distribution (Wood, Pya \& Säfken 2016). The scaled $t$-distribution adds two further parameters to the model, a scaling parameter $\sigma$ (estimated at 6.18) and a parameter for the degrees of freedom $v$ of the $t$-distribution (estimated at 0.29). Thus, for the present data, the residual error is characterized by $\epsilon / 6.18 \sim t_{(0.29)}$. Table 7 and Figures $1-3$ are based on this model.

As the present model is the result of exploratory data analysis, the $p$-values in Table 7, which all provide strong support for model terms with NDL measures as predictors, cannot be interpreted as the long-run probability of false positives. One might apply a stringent Bonferroni correction, and we note here that the large $t$-values for NDL model terms easily survive a correction for 1000 or even 10000 tests. However, we prefer to interpret the $p$-values simply as a measure of surprise 


\begin{tabular}{lrrrr}
\hline A. Parametric coefficients & Estimate & Std. error & $t$-Value & $p$-Value \\
\hline Intercept & -2.9179 & 0.2294 & -12.7173 & $<0.0001$ \\
Preceding = fricative & -0.0962 & 0.0299 & -3.2151 & 0.0013 \\
Preceding = nasal & -0.1335 & 0.0233 & -5.7229 & $<0.0001$ \\
Preceding = plosive & -0.1869 & 0.0150 & -12.4229 & $<0.0001$ \\
Preceding = vowel & 0.0106 & 0.0144 & 0.7318 & 0.4643 \\
Following = approximant & 0.2839 & 0.1470 & 1.9315 & 0.0534 \\
Following = fricative & 0.1036 & 0.1470 & 0.7048 & 0.4809 \\
Following = nasal & 0.1089 & 0.1474 & 0.7390 & 0.4599 \\
Following = plosive & 0.0850 & 0.1469 & 0.5785 & 0.5629 \\
Following = vowel & 0.1310 & 0.1469 & 0.8919 & 0.3725 \\
LocalSpeakingRate & -0.0463 & 0.0211 & -2.1874 & 0.0287 \\
IndividualSpeakingRate & 2.3873 & 0.6633 & 3.5990 & 0.0003 \\
\hline B. Smooth terms & edf & Ref.df & $F$-value & $p$-Value \\
\hline te(ActFromBoundaryDiphone, & & & & $<0.00001$ \\
ActDivFromBoundaryDiphone) & 14.4458 & 16.9557 & 548.4375 & $<0.0001$ \\
te(ActFromRemainingCues, & & & & $<0.0001$ \\
ActDivFromRemainingCues, & & & & \\
LocalSpeakingRate) & 24.7081 & 32.1035 & 170.9787 & $<0.0001$ \\
s(PriorMorph) & 2.0235 & 2.3027 & 84.2267 & $<0.0001$ \\
Random intercepts speaker & 37.1278 & 38.0000 & 2118.9174 & $<0.0001$ \\
Random intercepts word & 458.5028 & 2280.0000 & 2190.5616 & $<0.0001$ \\
\hline & & & & \\
\hline
\end{tabular}

Table 7

Summary of parametric and smooth terms in the generalized additive mixed model fit to the log-transformed acoustic duration of $\mathrm{S}$ as pronounced in the Buckeye corpus. The reference level for the preceding and following manner of articulation is 'absent'.

and an informal point measure of the relative degree of uncertainty about the parameter estimates.

Figure 1 presents the partial effect of PRIORMORPH. Larger priors go together with longer durations. This effect levels off slightly for larger priors. Apparently, inflectional lexomes with a stronger baseline activation tend to be articulated with longer durations. The $95 \%$ confidence interval (or more precisely, as GAMMs are empirical Bayes, the $95 \%$ credible interval) is narrow, especially for predictor values between 5 and 25, where most of the data points are concentrated.

Recall that PRIORMORPH has nine different values, one for each inflectional function of S. It is noteworthy that when we replace PRIORMORPH by a factor with the nine morphological functions as its levels, the model fit decreases (by 10 ML-score units), while at the same time the number of parameters increases by 7. The NDL prior for the inflectional functions, just by itself, already provides more 


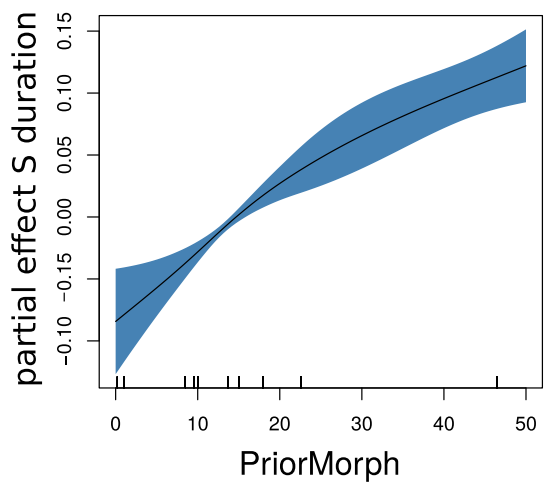

Figure 1

Partial effect of PRIORMORPH in the GAMM fit to S duration, with $95 \%$ confidence (credible) interval.

precision for predicting the duration of English S. Further precision is gained by also considering the activation and activation diversity measures.

Figure 2 presents the partial effect of the interaction of ACTFROMBOUNDARYDipHONE and ACTDIVFromBoundARYDIPHONE, which we modeled with a tensor product smooth. The left panel presents the contour lines with 1SE confidence intervals; the right panel shows the corresponding contour plot in color to facilitate interpretation, with darker shades of blue indicating shorter $\mathrm{S}$ and warmer yellow colors denoting longer $\mathrm{S}$. The narrow confidence bands in the left
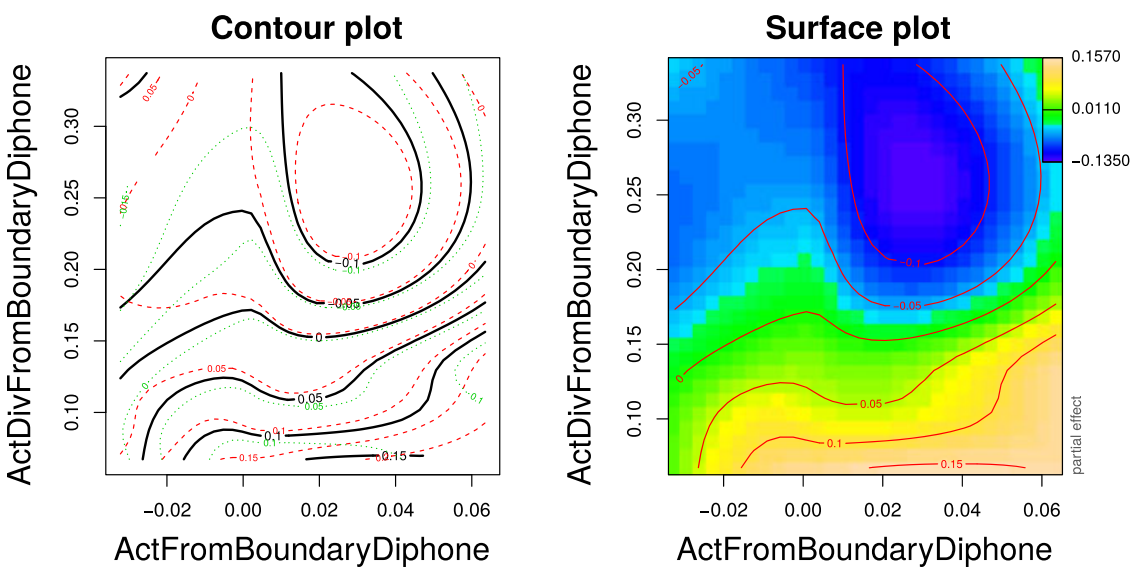

Figure 2

Partial effect in the GAMM fit to log-transformed $\mathrm{S}$ duration of the activation and activation diversity of the boundary diphone. In the right plot, deeper shades of blue indicate shorter acoustic durations and warmer shades of yellow denote longer durations. The left plot presents contour lines with 1SE confidence bands. 


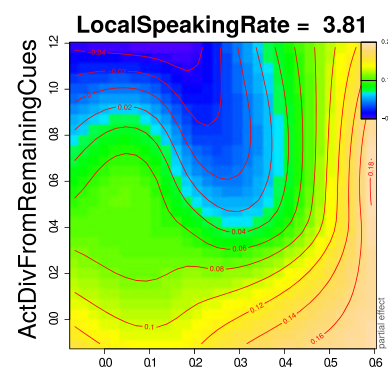

ActFromRemainingCues

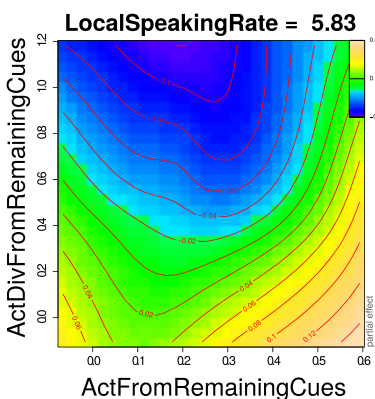

ActFromRemainingCues
LocalSpeakingRate $=4.67$

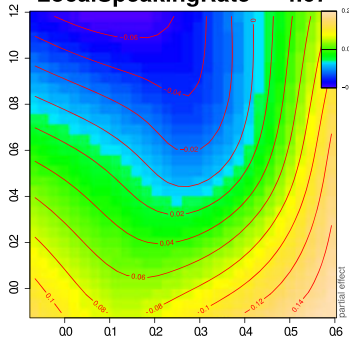

ActFromRemainingCues

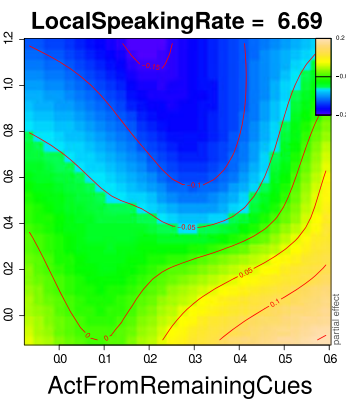

Figure 3
LocalSpeakingRate $=\mathbf{5 . 2 5}$

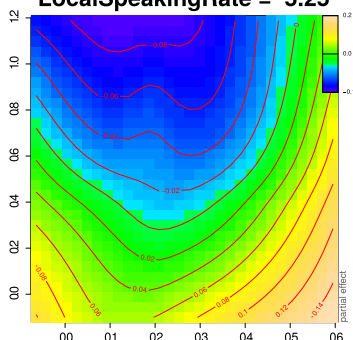

ActFromRemainingCues

Tensor product smooth for the three-way interaction of ACTFROMREMAININGCUES by ACTDIVFROMREMAININGCUES by local speaking rate. The regression surface for the two activation measures is shown for deciles $0.1,0.3,0.5,0.7$, and 0.9 of local speaking rate. Deeper shades of blue indicate shorter acoustic durations and warmer shades of yellow denote longer durations.

panel indicate that there are real gradients in this regression surface, except for the upper left corner of the plotting region. For all activation values, we find that as the activation diversity increases, $\mathrm{S}$ duration decreases. Conversely, for most values of activation diversity, increasing the activation leads to larger $\mathrm{S}$ duration. Shortest $\mathrm{S}$ durations are found for larger (but not the largest) values of activation and for activation diversities exceeding 0.2 . The two boundary measures interact insofar as $\mathrm{S}$ duration is strongly reduced for high DIVLASTDIPHONE in spite of high ActLastDiphone, as can be seen by the lake-like blue dip in the upper right quadrant of the plot. While smaller activation - and consequently reduced support - for the morphological function of $S$ should result in shorter $S$, it seems as though greater certainty about the morphological function counterbalances the trend, resulting in longer $\mathrm{S}$ (bottom left quadrant of the plot).

Figure 3 visualizes the three-way interaction of ACTFROMREMAININGCUES by ACTDIVFROMREMAININGCUES by local speaking rate. ${ }^{9}$ The successive

[9] Software for plotting confidence bands for these complex interactions is not available. 
panels of Figure 3 present the odd deciles of local speaking rate $(0.1,0.3,0.5,0.7$, and 0.9). The regression surface slowly morphs from one with long durations for high ACTDivFromREmAiningCuES (left panel) to a surface with long durations only in the lower right corner. The general pattern for ACTDIVFROMREMAININGCUES is that $\mathrm{S}$ duration decreases as ACTDIVFROMREMAININGCUES increases. For the lowest two deciles of local speech rate, this effect is absent for high values of ACTFRomREMAININGCUES. For ACTFromREMAININGCUES, we find that for lower values of ACTDIVFROMREMAININGCUES, durations increase with activation. For higher activation diversities, this effect is U-shaped. The interaction pattern between the two NDL measures mirrors the one found in Figure 2.

\section{Discussion}

\subsection{Summary of the present results}

Plag et al. (2017) reported that there are significant differences in the duration of English $\mathrm{S}$ as a function of the inflectional function realized by this exponent (see also Zimmermann 2016a, Seyfarth et al. 2018). Plag et al. (2017) observed that these differences in acoustic duration challenge the dominant current theories of morphology. These theories, which have their roots in post-Bloomfieldian American structuralism, hold that the relation between form and meaning in complex words is best understood in terms of a calculus in which rules operate on bound and free morphemes as well as on phonological units such as syllables and feet. However, the units of this theory, the configurations of these units, or the rules operating on these units or ensembles thereof cannot explain the observed differences in the duration of English $\mathrm{S}$ in an insightful way.

The present study explored whether the different durations of $\mathrm{S}$ can be understood as following from the extent to which words' phonological and collocational properties can discriminate between the inflectional functions expressed by the $\mathrm{S}$. We quantified the discriminability of these inflectional functions with three measures derived from a wide learning discrimination network that was trained on the entire Buckeye corpus. The input features (cues) for this network were words' lexomes in a five-word window centered on the S-bearing word and the diphones in the phonological forms of these lexomes. The classes to be predicted from these cues (the outcomes) were the inflectional functions (inflectional lexomes) of the $\mathrm{S}$.

Three measures derived from the network were predictive for the duration of S. A greater activation of a word's inflectional lexome (i.e. greater bottom-up support) predicted longer durations. A higher lexomic prior (i.e. a higher baseline activation or, equivalently, a higher degree of entrenchment in the network) also predicted longer durations. Apparently, both the support for a word's morphological function that is provided by that word's form and its collocational patterning as well as the a priori baseline support for the word that accumulates over the 
course of learning give rise to a prolonged acoustic signal. In other words, stronger support, both long-term and short-term, for a morphological function leads to an enhanced signal.

This finding dovetails well with lengthening of interfixes in Dutch, enhancement of English suffixes when they are paradigmatically more probable, and enhancement of vowels in Russian in proportion to paradigmatic support (Kuperman et al. 2007, Cohen 2014a, b, 2015). Signal enhancement as a function of activation also replicates the findings of Tucker et al. (2019) for the stem vowel of regular verbs in the Buckeye corpus.

The study by Tucker et al. (2019) reported an opposing force on the duration of verbs' stem vowels: the activation diversity. Activation diversity is a measure of lexicality. It assumes high values when the cues in the input are linked to many different outcomes. In such a case, the outcome is located in a dense lexicosemantic subspace and it is more difficult to discriminate the targeted outcome from its competitors. For auditory comprehension, we thus find that processing is slowed when activation diversity is high (Arnold et al. 2017). The flip side of the same coin is that in speech production, the prolonging part of the acoustic signal, such as $\mathrm{S}$, is dysfunctional when this signal increases the discrimination problem. A signal that is not discriminable cannot be made more discriminable by prolonging it. The prolongation will result only in lengthening a state of uncertainty instead of contributing to resolving it. Importantly, a large activation diversity is dysfunctional not only for the listener but also for the speaker. The auditory image that the speaker projects and aims to realize through articulation (Hickok 2014) feeds back through the control loop to the semantic system. As a consequence, aspects of the speech signal that are problematic for the listener will also be problematic for the speaker.

Considered together, the three NDL measures indicate that the speaker has to balance two opposing forces. One force seeks to lengthen parts of the signal in the presence of strong bottom-up support and long-term expectations. The other force seeks to shorten parts of the signal that increase uncertainty. The NDL measures enable us to probe these forces. More importantly, our model illustrates that these two forces interact in an unexpected way. In case one force creates extreme uncertainty about the morphological function of $\mathrm{S}$, the other force is able to reduce this uncertainty and $\mathrm{S}$ durations turn out to be long.

The framework of naïve discriminative learning accepts that the language system is, to some degree, 'chaotic'. Just as in weather systems, a butterfly flapping its wings in the Amazon is claimed to be able to start a chain of events that cause a rainstorm in London (Lorenz 1972), the cues that co-occur across learning events with cues that go together with a target word can co-determine the discriminability of that target word; see Mulder et al. (2014) for an interpretation of the secondary family size effect along these lines.

Thus, the approach presented in the current study - training of an NDL network that learns to discriminate linguistic outcomes on the basis of sublexical and collocational properties and deriving NDL measures from this network to predict 
the acoustic properties of a linguistic item - can be adopted to investigate similar phenomena in other languages. For example, we are currently investigating how the different inflectional and derivational functions of final /s/ and final schwa in Dutch affect their acoustic durations. The approach allows one to investigate the lexical structure of not only morphological functions but maybe even semantic contrasts. Given the present results as well as those by Tucker et al. (2019), this approach is very promising.

\subsection{Consequences for morphological theory}

The question remains as to whether this 'chaotic' explanation of non-random variation in $\mathrm{S}$ duration improves on an explanation that simply posits that different morphological functions have different consequences for $\mathrm{S}$ duration. Rephrased statistically, does the prediction accuracy increase when we replace a model with a factor for morphological function (with nine levels) with a model in which this factor is replaced with NDL measures? When we replace the factor inflection type by just the NDL prior, a numeric variable with nine distinct values, model fit indeed improves, while at the same time model complexity decreases. Instead of needing eight parameters for inflectional function, only a single parameter (the slope of the regression line) suffices. When the linearity assumption for the prior is relaxed, the required effective degrees of freedom is still well below 8 .

What are the consequences of our findings for morphological theory and theories of speech production? First, consider morphological theory. Here, we are confronted with a range of different approaches that rest on very different assumptions about the structure of words. Two major approaches are relevant in the context of the S problem. On the one hand, we have post-Bloomfieldian itemand-arrangement theories (IAA; Hockett 1954) and generative offshoots thereof building on Chomsky \& Halle (1968). On the other hand, we have realizational theories such as word and paradigm morphology (WP) (Blevins 2006). Both WP and IAA address how inflectional functions such as number and tense are expressed in speech. IAA posits that this expression is mediated by morphemes, i.e. the minimal units of a language that combine form and meaning. WP, on the other hand, rejects the usefulness of the morpheme as a theoretical construct (see also Matthews 1974, Beard 1977, Aronoff 1994, Blevins 2003). Instead of constructing a calculus for building words out of morphemes, WP focuses on the paradigmatic relations between words and holds that morphological systematicities are driven by certain paradigm-internal mechanisms, e.g. proportional analogy. Naïve discriminative learning provides a computational modeling framework that is deeply influenced by WP morphology, and the measures derived from the model can be understood as gauging aspects of proportional analogies. The specific implementation proposed in this study of English S extends proportional analogy by including 'collocational analogy' along with phonological analogy. For a detailed discussion of proportional analogy and discriminative learning, see Baayen et al. (2019). 
It is less clear whether the present findings are compatible with IAA. Explanations within IAA can attribute an effect to representations for units, to configurations of such units, as well as to the combinatorial rules that give rise to these configurations. Plag et al. (2017) showed that the observed differences in the durations of English $\mathrm{S}$ cannot be explained in this way. However, IAA can assign conditional probabilities to units and configurations of units and link the likelihood of an effect to such probabilities (see, among others, Jurafsky et al. 2000, Aylett \& Turk 2004, Gahl 2008, Bell et al. 2009, Tremblay \& Tucker 2011, Cohen Priva 2015, Kleinschmidt \& Jaeger 2015). We cannot rule out that probabilities for inflectional functions that are properly conditioned on collocational and phonological distributional patterns will also predict the duration of English S. In the light of previous studies (Milin et al. 2017b, Tucker et al. 2019), however, we anticipate that such measures will underperform compared to discriminative measures. We note here that if measures such as, for instance, the probability of a genitive plural conditioned on the two preceding and following words are indeed found to be effective predictors of $\mathrm{S}$ duration, this would imply that the fine-tuning of the duration of $\mathrm{S}$ takes place after morphemes have been assembled into phrases. In other words, any fine-tuning of this kind must, within the generative framework, take place post-lexically.

\subsection{Implications for speech production}

Having outlined the implications of our findings for theoretical morphology, we next consider their implications for models of speech production. The literature on speech production is dominated by two models, those of Dell (1986) and Levelt et al. (1999). Both models take the framework of IAA as given and propose mechanisms for assembling from morphemes and phonemes the form representations posited to drive articulation.

Dell's interactive activation model is set up in such a way that the activation of morphemes can be influenced by other words in the phrase. The paradigmatic effect of activation diversity, which we calculated for all inflectional functions that can be realized as $\mathrm{S}$, however, cannot be captured by this model, as in most phrases only one, perhaps two, of these inflectional functions are relevant. It is also unclear how effects of the NDL prior might be accounted for, as the model does not implement baseline activation levels. Furthermore, the activation measure in our learning model integrates evidence from all words in the five-word window to the $\mathrm{S}$, whereas in Dell's model, inflectional morphemes receive activation only from an inflectional concept node.

The WEAVER model by Levelt et al. implements a strictly modular architecture, with a lemma layer separating morphemes from concepts. In this model, the selection of the stem is handled by hard-wired links between lemmas' word forms one layer down in the model's hierarchy. The selection of a specific inflectional morpheme is driven by diacritical features associated with a word's lemma. Whether an inflectional suffix is selected depends on whether its corresponding 
diacritical feature is flagged as active. Since WEAVER explains frequency effects at the word form level, it might be possible to interpret the inflectional priors from the NDL network as the resting activation levels of the inflectional morphemes in WEAVER's form stratum. However, since the WEAVER model is not a learning model, each of the nine values of the NDL prior unavoidably becomes free parameters of the model. Furthermore, the way the priors are estimated in our NDL model, namely, by evaluating entrenchment across all diphones and even the words in the immediate context, is completely at odds with WEAVER's modular design. Since WEAVER's design precludes the possibility of neighborhood similarity effects - a prediction that has been shown to be incorrect (Vitevitch 2002, Vitevitch \& Stamer 2006, Vitevitch 2008, Scarborough 2004) - it is unlikely that this model can be adapted to integrate discriminative information across the full lexicon.

After all, we are predicting the amounts of support for inflectional diacritics, and one could imagine that our effects could be captured by some decision process selecting the proper diacritic that then drives standard weaver production. In fact, the positive correlations of prior and activation with $\mathrm{S}$ duration run counter to the predictions of information theoretic accounts and probabilistic theories building on IAA models, according to which words and segments are realized shorter when they are less informative (Aylett \& Turk 2004, Jaeger 2010, Cohen Priva 2015). However, our results dovetail well with the Paradigmatic Signal Enhancement Hypothesis (Kuperman et al. 2007), which holds that the more probable an exponent is in a given paradigm, the longer it will be articulated (see also Ernestus \& Baayen (2006) and Cohen (2014a)). Kuperman et al. observed that the duration of an interfix in Dutch compounds was proportional to its probability within the left constituent family of the compound. For English S, it is the set of inflectional lexomes that $\mathrm{S}$ realizes that constitutes the paradigm within which both support and uncertainty are evaluated.

\subsection{NDL as a computational tool of speech production}

This survey leaves us with the question of how to understand NDL as a computational model and especially its status as a computational model of speech production. After all, the network that we have found to predict $S$ duration best represents the comprehension part of an internal production-comprehensionproduction feedback loop. To answer this question, we first note that NDL is a computational tool, very similar to multiple regression (Evert \& Arppe 2016, Sering et al. 2018b), which helps us trace the consequences of low-level, implicit statistical learning. It affords prediction precision that is not available to handcrafted architectures such as that found in the models of Dell and Levelt and colleagues. Second, because NDL is a computational tool, it does not provide a full model of either comprehension or production. Nevertheless, applied to English S, this tool informs us that, surprisingly, it is the comprehension part of the feedback loop that appears to be crucial for understanding the variance in $S$ 
duration. Furthermore, if the way in which the model is set up, with lexomic and phonological cues jointly predicting inflectional lexomes, is on the right track, this challenges separation of processes into lexical and post-lexical. The reason is that during learning, phonological and lexomic cues are in competition in a fundamentally non-modular way.

A more complete, and unavoidably more modular, model for the mental lexicon that integrates comprehension and production was recently proposed by Baayen, Baayen \& Blevins (2018) and Baayen et al. (2019). Their model, linear discriminative learning (LDL), comprises several interacting networks, with modality-specific numeric vectors for words' forms and with semantic vectors that replace the lexomic pointers that stand in for words' meanings in naïve discriminative learning. For inflected words, these semantic vectors are obtained by summing the semantic vectors of the lexomes realized in that word. Thus, the semantic vector for $\operatorname{dog} s, \overrightarrow{\operatorname{dog} s}$, is $\overrightarrow{\operatorname{dog}}+\overrightarrow{\operatorname{dogs}}$. The production part of the LDL model first maps a word's semantic vector onto a numeric vector representing that word's form, using a straightforward linear transformation (i.e. a two-layer linear network). The length of words' form vectors is equal to the number of different n-phones known to the model, and the values in these vectors specify the degree to which the different n-phones are supported by the words' semantic vectors. Once a form vector has been obtained, the model calculates the set of ordered sequences of overlapping n-phones (e.g. \#do+dog+ogz+gz\#) that are best supported by the form vector. For each of the resulting candidates for production, the model then calculates how well these candidates succeed in realizing the intended meaning when presented to the model's comprehension network. The pertinent calculations again make use of a linear transformation but, now, one that maps form vectors onto semantic vectors. The candidate with the semantic vector closest to that of the original semantic vector targeted for production is selected for articulation. Thus, in this model, there is an explicit feedback loop from form to meaning. Measures based on this model that are expected to be predictive for $S$ duration are the amount of support from the semantics for the pertinent n-phones, the angles and distances between the semantic vectors of inflectional lexomes, and the angles or distances between these inflectional vectors and the semantic vectors generated by the feedback loop. We leave the exploration of such measures for further research, given that NDL provides simple yet effective measures gauging the support of form for meaning. ${ }^{10}$

[10] We note here that neither NDL nor LDL provide fully specified, 'mechanical' models. In particular, the mechanisms that are responsible for selection processes are not implemented. As a consequence, we have to rely on generalized additive models to chart the details of the interplay of the network support and decision processes. 


\subsection{Conclusion}

We conclude with placing the present findings in a broader perspective. Speakers tend to smooth articulatory gestures across junctures, resulting in a variety of forms of assimilation. Simplification of articulatory gestures can give rise to substantial reduction of spoken words compared to dictionary norms (Ernestus 2000, Johnson 2004, Ernestus, Baayen \& Schreuder 2002, Arnold et al. 2017). How exactly words are realized in speech depends on the interplay of many factors, including audience design (Lindblom 1990), minimization of effort (Zipf 1949), information density management (Aylett \& Turk 2004, Jaeger 2010, Bell et al. 2009), articulatory proficiency (Tomaschek et al. 2018c, a, 2019), speech rhythm (Ernestus \& Smith 2018), and paradigmatic enhancement (Kuperman et al. 2007, Cohen 2014a). To this list, the present study adds 'discrimination management' for inflectional functions (see also Tucker et al. 2019). When an exponent such as $\mathrm{S}$ provides strong support for the targeted inflectional lexome (gauged by NDL activations and priors), it is articulated with longer duration. When $\mathrm{S}$ fails as a discriminative cue and instead creates uncertainty about the intended inflectional function, its duration is decreased. Energy is not invested in a signal that creates confusion instead of clarity.

The current study shows that the discriminative capability of single segments as well as parts of the speech signal affect its acoustic characteristics. Discriminative capability is similar to what Wedel, Kaplan \& Jackson (2013b) call 'functional load', i.e. the number of words a single segment discriminates. Using counts of minimal pairs, Wedel and colleagues have shown that a high functional load inhibits the loss of phonological contrasts (see also Wedel, Jackson \& Kaplan 2013a). Hall et al. (2018) further argue that phonetic segments get enhanced when there is a risk to misidentify one word as another one (e.g. the [p] in pill is longer than that in pipe because it has to be contrasted more strongly from bill, whereas there is no *bipe). This argumentation is consistent with our findings. A greater activation for a morphological function indicates that the cues in the context of the S-bearing word contrast the morphological function very well from other morphological functions. A lower activation diversity indicates that the morphological function can be well contrasted on the basis of the contextual cues in the signal. In both cases, we observe an enhancement of the signal, namely lengthening.

The measures derived from naïve discriminative learning offer the researcher new tools that probe language structure at a much more fine-grained level than is possible with minimal pairs. Thanks to these tools, we can now begin to further improve our understanding of how discriminative capability modulates segment duration. 


\section{APPENDIX A. NDL: RESCORLA-WAGNER EQUATIONS}

Technically, the Rescorla-Wagner equations are closely related to the perceptron (Rosenblatt 1962) and adaptive learning in electrical engineering (Widrow \& Hoff 1960). The Rescorla-Wagner equations estimate the association strength, henceforth weights $\mathcal{W}$, between input units $\mathcal{C}$, with $\mathcal{C} \in c_{k}, k=1,2, \ldots, K$, henceforth cues, and a set of output units $\mathcal{O}$, with $\mathcal{O} \in o_{n}, n=1,2, \ldots, N$, henceforth outcomes.

During learning, each outcome $\mathcal{O}_{j}$ is defined by a set of cues, henceforth cue set $\mathcal{C S}_{\Omega}$. Usually, $q$ equals $j$. Since $j$ also represents the position of $\mathcal{O}$ in the weight matrix, we use $q$ as a pointer to the associated $\mathcal{O}_{j}$.

The size of the weight matrix increases incrementally during learning whenever new subsets of cues and outcomes are encountered. After training, the RescorlaWagner network will be defined by a $K \times N$ weight matrix, where $K$ represents the total number of unique cues encountered during learning and $N$ represents the total number of encountered unique outcomes during learning.

At a given learning event $L_{t}, t=1,2, \ldots, T$, weights are adapted on the connections from the inputs present during the learning event $t$, henceforth the cues $\mathcal{C}_{t}\left(\mathcal{C}_{t} \subseteq C\right)$, to all of the outcomes $\mathcal{O}_{1, \ldots, t}$ that have been encountered at least once during any of the learning events $1,2, \ldots, t-1$. The outcomes present at learning event $L_{t}$ are denoted by $\mathcal{O}_{t}\left(\mathcal{O}_{t} \subseteq \mathcal{O}\right)$. The weight between cue $c_{i}$ and outcome $o_{j}$ at the end of the learning event $t$ is given by

$$
w_{i j}^{(t)}=w_{i j}^{(t-1)}+\Delta w_{i j}^{(t-1)},
$$

$\Delta w_{i j}^{t-1}$ is calculated by the Rescorla-Wagner equations:

$$
\Delta w_{i j}^{(t-1)}= \begin{cases}\text { (a) } 0 & \text { if } c_{i} \notin c_{t}, \\
\text { (b) } \alpha_{i} \beta_{j}\left(\lambda-\sum_{m} \mathrm{I}_{\left[c_{m} \in \mathcal{C}_{t}\right]} w_{m j}^{(t-1)}\right) & \text { if } c_{i} \in \mathcal{C}_{t} \wedge o_{j} \in \mathcal{O}_{j}, \\
\text { (c) } \alpha_{i} \beta_{j}\left(0-\sum_{m} \mathrm{I}_{\left[c_{m} \in \mathcal{C}_{t}\right]} w_{m j}^{(t-1)}\right) & \begin{array}{r}
\text { if } c_{i} \in \mathcal{C}_{t} \wedge o_{j} \notin \mathcal{O}_{j} \\
\wedge o_{j} \in \mathcal{O}_{1, \ldots, t-1}, \\
\text { (d) } 0
\end{array} \\
\text { otherwise. }\end{cases}
$$

The Rescorla-Wagner equations define four conditions which define adaptation strength $\Delta w_{i j}^{(t-1)}$ on the efferent weights in learning event $t$. The maximum learnability, $\lambda$, was set to 1.0 in all our calculations, while cue and outcome salience, $\alpha_{i}$ and $\beta_{j}$, were set to 0.1. The four conditions in Equation (A2) define the following states:

1. if the $i$ th cue is not an element of the active cues $C_{t}$ during the event $L_{t}$, $\Delta w_{i j}^{(t-1)}$ is equal to zero and none of its efferent weights are adapted. 
2. If the $i$ th cue is an element of the active cues in a learning event $C_{t}$, the connection to $o_{j}$ is strengthened if $o_{j}$ is also present in the event $t$ by subtracting the sum of the weights across all cues in $C_{t}$ from $\lambda$. As a result, $\Delta w_{i j}^{t-1}$ is inversely proportional to the number of present cues. $I$ is the indicator operator, which evaluates to 1 if its argument in square brackets is true, and to zero otherwise. $m$ indexes the cues in $C_{t}$.

3. If $o_{j}$ is not present but has been encountered during some previous learning event, the strength of the connection between $c_{i}$ weight and $o_{j}$ is reduced by subtracting the sum of the weights across all cues in $C_{t}$ from 0 . As a result, $\Delta w_{i j}^{t-1}$ is proportional to the number of present cues.

4. If none of the above three conditions is true, $\Delta w_{i j}^{(t-1)}$ is equal to zero. This is especially the case when an outcome is encountered which was not present in any of the previous learning events.

\section{REFERENCES}

Arnold, D., F. Tomaschek, F. Lopez, Tino Sering \& R. H. Baayen. 2017. Words from spontaneous conversational speech can be recognized with human-like accuracy by an error-driven learning algorithm that discriminates between meanings straight from smart acoustic features, bypassing the phoneme as recognition unit. PLOS ONE 12.4, e0174623. http://journals.plos.org/plosone/article? id=10.1371/journal.pone. 0174623 .

Aronoff, M. 1994. Morphology by itself: Stems and inflectional classes. Cambridge, MA: The MIT Press.

Aylett, M. \& A. Turk. 2004. The smooth signal redundancy hypothesis: A functional explanation for relationships between redundancy, prosodic prominence, and duration in spontaneous speech. Language and Speech 47, 31-56.

Baayen, R. H., P. Milin, D. Filipović Durdević, P. Hendrix \& M. Marelli. 2011. An amorphous model for morphological processing in visual comprehension based on naïve discriminative learning. Psychological Review 118, 438-482. doi:10.1037/a0023851.

Baayen, R. H., P. Milin \& M. Ramscar. 2016a. Frequency in lexical processing. Aphasiology 30.11, 1174-1220. doi:10.1080/02687038.2016.1147767.

Baayen, R. H., C. Shaoul, J. Willits \& M. Ramscar. 2016b. Comprehension without segmentation: A proof of concept with naïve discriminative learning. Language, Cognition, and Neuroscience 31.1, 106-128. doi:10.1080/23273798.2015.1065336.

Baayen, R. Harald, Yu-Ying Chuang \& James P. Blevins. 2018. Inflectional morphology with linear mappings. The Mental Lexicon 13.2, 230-268.

Baayen, R. Harald, Yu-Ying Chuang, Elnaz Shafaei-Bajestan \& James P. Blevins. 2019. The discriminative lexicon: A unified computational model for the lexicon and lexical processing in comprehension and production grounded not in (de) composition but in linear discriminative learning. Complexity. Article ID 4895891, 39 pages doi:10.1155/2019/4895891.

Bates, D. M., M. Mächler, B. Bolker \& S. Walker. 2015. Fitting linear mixed-effects models using lme4. Journal of Statistical Software 67.1, 1-48. doi:10.18637/jss.v067.i01.

Beard, R. 1977. On the extent and nature of irregularity in the lexicon. Lingua 42, 305-341.

Bell, A., D. Jurafsky, E. Fosler-Lussier, C. Girand, M. Gregory \& D. Gildea. 2003. Effects of disfluencies, predictability, and utterance position on word form variation in English conversation. Journal of the Acoustical Society of America 113, 1001-1024.

Bell, Alan, Jason M. Brenier, Michelle Gregory, Cynthia Girand \& Dan Jurafsky. 2009. Predictability effects on durations of content and function words in conversational English. Journal of Memory and Language 60.1, 92-111.

Ben Hedia, Sonia \& Ingo Plag. 2017. Gemination and degemination in English prefixation: Phonetic evidence for morphological organization. Journal of Phonetics 62, 34-49.

Bergmann, Pia. 2015. Morphologisch komplexe Wörter im Morphologisch komplexe Wörter im Deutschen: Prosodische Struktur und phonetische Realisierung: Habilitationsschrift, AlbertLudwigs-Universität Freiburg. 
Bermúdez-Otero, Ricardo. 2018. Stratal phonology. In S. J. Hannahs \& Anna Bosch (eds.), Routledge handbook of phonological theory, 100-134. London, UK: Routledge.

Blazej, Laura J. \& Ariel M. Cohen-Goldberg. 2015. Can we hear morphological complexity before words are complex? Journal of Experimental Psychology. Human Perception and Performance 41.1, 50-68.

Blevins, James P. 2003. Stems and paradigms. Language 79, 737-767.

Blevins, James P. 2006. Word-based morphology. Journal of Linguistics 42.03, 531-573.

Blevins, James P., Farrell Ackerman \& Robert Malouf. 2016. Morphology as an adaptive discriminative system. In Heidi Harley \& Daniel Siddiqi (eds.), Morphological metatheory, 271-301. Amsterdam and Philadelphia: John Benjamins.

Bybee, Joan L. 2001. Phonology and language use. Cambridge: Cambridge University Press.

Chomsky, N. \& M. Halle. 1968. The sound pattern of English. New York: Harper and Row.

Cohen, Claire. 2014a. Combining structure and usage patterns in morpheme production: Probabilistic effects of sentence context and inflectional paradigms. Berkeley: University of California $\mathrm{PhD}$ dissertation.

Cohen, Clara. 2014b. Probabilistic reduction and probabilistic enhancement. Morphology 24.4, 291-323.

Cohen, Clara. 2015. Context and paradigms: Two patterns of probabilistic pronunciation variation in Russian agreement suffixes. Mental Lexicon 10.3, 313-338. doi:10.1075/ml.10.3.01coh. http:// eprints.gla.ac.uk/133559/.

Cohen Priva, U. 2015. Informativity affects consonant duration and deletion rates. Laboratory Phonology 6.2, 243-278.

Dell, G. S. 1986. A spreading-activation theory of retrieval in sentence production. Psychological Review 93, 283-321.

Drager, Katie. 2011. Sociophonetic variation and the lemma. Journal of Phonetics 39.4, 694-707.

Ernestus, M. 2000. Voice assimilation and segment reduction in casual Dutch. A corpus-based study of the phonology-phonetics interface. Utrecht: LOT.

Ernestus, M., R. H. Baayen \& R. Schreuder. 2002. The recognition of reduced word forms. Brain and Language 81, 162-173. doi:10.1006/brln.2001.2514.

Ernestus, Mirjam \& R. H. Baayen. 2006. The functionality of incomplete neutralization in Dutch. The case of past-tense formation. Laboratory Phonology 8, 27-49.

Ernestus, Mirjam \& R. Harald Baayen. 2011. Corpora and exemplars in phonology. The handbook of phonological theory, 2nd edn. 374-400. Wiley-Blackwell.

Ernestus, Mirjam \& Rachel Smith. 2018. Qualitative and quantitative aspects of phonetic variation in Dutch eigenlijk. In Francesco Cangemi, Meghan Clayards, Oliver Niebuhr, Barbara Schuppler \& Margaret Zellers (eds.), Rethinking reduction. Berlin-New York: De Gruyter.

Evert, S. \& A. Arppe. 2016. Some theoretical and experimental observations on naive discriminative learning x. In Johannes Wahle, Marisa Koellner, Harald R. Baayen, Gerhard Jaeger \& Tineke Baayen-Oudshoorn (eds.), Proceedings of 6th Conference Quantitative Investigations in Theoretical Linguistics (QITL-6). Tübingen: Universität Tübingen.

Foulkes, Paul, Gerard Docherty \& Dominic Watt. 2005. Phonological variation in child-directed speech. Language 81.1, 177-206.

Gahl, Susanne. 2008. Time and thyme are not homophones: The effect of lemma frequency on word durations in spontaneous speech. Language 84.3, 474-496.

Goad, Heather. 1998. Plurals in SLI: Prosodic deficit or morphological deficit? Language Acquisition 7.2-4, 247-284.

Goad, Heather, Lydia White \& Jeffrey Steele. 2003. Missing inflection in L2 acquisition: Defective syntax or L1-constrained prosodic representations? The Canadian Journal of Linguistics/La Revue Canadienne de Linguistique 48.2, 243-263.

Hall, Kathleen Currie, Elizabeth Hume, T. Florian Jaeger \& Andrew Wedel. 2018. The role of predictability in shaping phonological patterns. Linguistics Vanguard 4(s2).

Hay, J. B. 2002. From speech perception to morphology: Affix-ordering revisited. Language 78, $527-555$.

Hay, J. B. 2003. Causes and consequences of word structure. New York and London: Routledge.

Hay, Jen B. \& R. H. Baayen. 2002. Parsing and productivity. In G. E. Booij \& J. Van Marle (eds.), Yearbook of morphology 2001, 203-235. Dordrecht: Kluwer Academic Publishers.

Hay, Jennifer. 2007. The phonetics of un-. In Judith Munat (ed.), Lexical creativity, texts and contexts, 39-57. Amsterdam/Philadelphia: Benjamins. 
Hickok, Gregory. 2014. The architecture of speech production and the role of the phoneme in speech processing. Language, Cognition and Neuroscience 29.1, 2-20.

Hockett, C. 1954. Two models of grammatical description. Word 10, 210-231.

Jaeger, F. 2010. Redundancy and reduction: Speakers manage syntactic information density. Cognitive Psychology 61, 23-62.

Johnson, K. 2004. Massive reduction in conversational American English. Spontaneous speech: data and analysis. Proceedings of the 1st Session of the 10th International Symposium, 29-54. Tokyo, Japan: The National International Institute for Japanese Language.

Jurafsky, D., A. Bell, M. Gregory \& W. D. Raymond. 2001a. Probabilistic relations between words: Evidence from reduction in lexical production. In J. L. Bybee \& P. Hopper (eds.), Frequency and the emergence of linguistic structure, 229-254. Amsterdam: Benjamins.

Jurafsky, Dan, Alan Bell, Michelle Gregory \& William D. Raymond. 2001b. The effect of language model probability on pronunciation reduction. Proceedings of the 2001 IEEE Conference on Acoustics, Speech, and Signal Processing, 801-804. Salt Lake City, UT, USA: IEEE.

Jurafsky, Daniel, Alan Bell \& Cynthia Girand et al. 2000. The role of the lemma in form variation. In Natasha Warner Carlos Gussenhoven (ed.), Papers in laboratory phonology vii, 3-34. Berlin: De Gruyter Mouton.

Kalman, R. E. 1960. A new approach to linear filtering and prediction problems. Journal of basic Engineering 82.1, 35-45.

Kamin, L. J. 1969. Predictability, surprise, attention, and conditioning. In B. A. Campbell \& R. M. Church (eds.), Punishment and aversive behavior, 276-296. New York: Appleton-Century-Crofts.

Keating, Patricia A. 2006. Phonetic encoding of prosodic structure. In Jonathan Harrington \& Marija Tabain (eds.), Speech production: Models, phonetic processes, and techniques, 167-186. New York: Psychology Press.

Kemps, Rachèl, Mirjam Ernestus, Robert Schreuder \& R. Harald Baayen. 2005a. Prosodic cues for morphological complexity: The case of Dutch plural nouns. Memory \& Cognition 33.3, 430-446. doi:10.3758/BF03193061.

Kemps, Rachèl J. J. K., Lee H. Wurm, Mirjam Ernestus, Robert Schreuder \& R. Harald Baayen. 2005b. Prosodic cues for morphological complexity in Dutch and English. Language and Cognitive Processes 20.1-2, 43-73.

Kiparsky, Paul. 1982. From cyclic phonology to lexical phonology. In Harry van der Hulst \& Norval Smith (eds.), The structure of phonological representations, 131-176. Dordrecht: Foris.

Kleinschmidt, Dave F. \& T. Florian Jaeger. 2015. Robust speech perception: Recognize the familiar, generalize to the similar, and adapt to the novel. Psychological Review 122.2, 148.

Kuperman, Victor, Mark Pluymaekers, Mirjam Ernestus \& Harald Baayen. 2007. Morphological predictability and acoustic salience of interfixes in Dutch compounds. JASA 121, 2261-2271.

Kuznetsova, Alexandra, Per Bruun Brockhoff \& Rune Haubo Bojesen Christensen. 2014. lmerTest. http://cran.r-project.org/web/packages/lmerTest/index.html.

Landauer, T. K. \& S. T. Dumais. 1997. A solution to Plato's problem: The latent semantic analysis theory of acquisition, induction and representation of knowledge. Psychological Review 104.2, 211-240.

Lee-Kim, Sang-Im, Lisa Davidson \& Sangjin Hwang. 2013. Morphological effects on the darkness of English intervocalic /1/. Laboratory Phonology 4.2, 475-511.

Levelt, William J. M. \& Linda R. Wheeldon. 1994. Do speakers have access to a mental syllabary. Cognition 50, 239-269.

Levelt, W. J. M., A. Roelofs \& A. S. Meyer. 1999. A theory of lexical access in speech production. Behavioral and Brain Sciences 22, 1-38.

Lindblom, B. 1990. Explaining phonetic variation: A sketch of the H\&H theory. In W. J. Hardcastle \& A. Marchal (eds.), Speech production and speech modeling, 403-440. Dordrecht: Kluwer.

Lohmann, Arne. 2018a. Cut (n) and cut (v) are not homophones: Lemma frequency affects the duration of noun-verb conversion pairs. Journal of Linguistics 54.4, 753-777.

Lohmann, Arne. 2018b. Time and thyme are not homophones: A closer look at gahl's work on the lemma-frequency effect, including a reanalysis. Language 94.2 , e180-e190.

Lorenz, E. 1972. Predictability. Paper presented at the 139th AAAS Meeting.

Losiewicz, Beth L. 1992. The effect of frequency on linguistic morphology. PhD dissertation. Austin, TX: University of Texas.

Lund, K. \& C. Burgess. 1996a. Producing high-dimensional semantic spaces from lexical cooccurrence. Behaviour Research Methods, Instruments, and Computers 28.2, 203-208. 
Lund, K. \& C. Burgess. 1996b. Producing high-dimensional semantic spaces from lexical cooccurrence. Behavior Research Methods Instruments and Computers 28.2, 203-208.

Marsolek, C. J. 2008. What antipriming reveals about priming. Trends in Cognitive Science 12.5, 176-181. doi:10.1016/j.tics.2008.02.005.

Matthews, P. H. 1974. Morphology. An introduction to the theory of word structure. London: Cambridge University Press.

Mikolov, Tomas, Ilya Sutskever, Kai Chen, Greg S. Corrado \& Jeff Dean. 2013. Distributed representations of words and phrases and their compositionality. In C. J. C. Burges, L. Bottou, M. Welling, Z. Ghahramani \& K. Q. Weinberger (eds.), Advances in neural information processing systems, 3111-3119. Electronic Proceedings of the Neural Information Processing Systems Conference.

Milin, P., D. Divjak \& R. H. Baayen. 2017a. A learning perspective on individual differences in skilled reading: Exploring and exploiting orthographic and semantic discrimination cues. Journal of Experimental Psychology: Learning, Memory, and Cognition. doi:10.1037/xlm0000410.

Milin, P., L. B. Feldman, M. Ramscar, P. Hendrix \& R. H. Baayen. 2017b. Discrimination in lexical decision. PLoS ONE 12.2, e0171935.

Mulder, K., T. Dijkstra, R. Schreuder \& R. H. Baayen. 2014. Effects of primary and secondary morphological family size in monolingual and bilingual word processing. Journal of Memory and Language 72, 59-84.

Nespor, Marina \& Irene Vogel. 2007. Prosodic phonology. Berlin, New York: Walter de Gruyter.

Pitt, Mark A., Laura Dilley, Keith Johnson, Scott Kiesling, William Raymond, Elizabeth Hume \& Eric Fosler-Lussier. 2007. Buckeye corpus of conversational speech (2nd release). Columbus, $\mathrm{OH}$ : Department of Psychology, Ohio State University.

Plag, Ingo. 2018. Word-formation in English, 2nd edn. Cambridge: Cambridge University Press.

Plag, Ingo. 2014. Homophony in morphology: The acoustic properties of morphemic and nonmorphemic word-final S in English. Plenary talk. 14th International Morphology Meeting, Budapest.

Plag, Ingo, Julia Homann \& Gero Kunter. 2017. Homophony and morphology: The acoustics of wordfinal $\mathrm{S}$ in English. Journal of Linguistics 53.1, 181-216.

Pluymaekers, M., M. Ernestus \& R. H. Baayen. 2005a. Articulatory planning is continuous and sensitive to informational redundancy. Phonetica 62, 146-159.

Pluymaekers, M., M. Ernestus \& R. H. Baayen. 2005b. Lexical frequency and acoustic reduction in spoken Dutch. Journal of the Acoustical Society of America 118.4, 2561-2569.

Podlubny, R. G., K. Geeraert \& B. V. Tucker. 2015. Its all about, like, acoustics. In The Scottish Consortium for ICPhS 2015 (ed.), Proceedings of the ICPHS IIXX. Glasgow, UK: The University of Glasgow.

Ramscar, M., M. Dye \& J. Klein. 2013a. Children value informativity over logic in word learning. Psychological Science 24.6, 1017-1023.

Ramscar, M., Melody Dye \& Stewart M. McCauley. 2013b. Error and expectation in language learning: The curious absence of mouses in adult speech. Language 89.4, 760-793. doi:10.1353/ lan.2013.0068.

Ramscar, M., Melody Dye, Hanna Muenke Popick \& Fiona O’Donnell-McCarthy. 2011. The enigma of number: Why children find the meanings of even small number words hard to learn and how we can help them do better. PloS ONE 6.7, e22501. doi:10.1371/journal.pone.0022501.

Ramscar, M., P. Hendrix, C. Shaoul, P. Milin \& R. H. Baayen. 2014. Nonlinear dynamics of lifelong learning: the myth of cognitive decline. Topics in Cognitive Science 6, 5-42. doi:10.1111/tops.120 78.

Ramscar, M., C. C. Sun, P. Hendrix \& R. H. Baayen. 2017. The mismeasurement of mind: Lifespan changes in paired-associate-learning scores reflect the 'cost' of learning, not cognitive decline. Psychological Science 28.8, 1171-1179. doi:10.1177/0956797617706393.

Ramscar, M. \& D. Yarlett. 2007. Linguistic self-correction in the absence of feedback: A new approach to the logical problem of language acquisition. Cognitive Science 31.6, 927-960. doi:10.1080/0364 0210701703576.

Ramscar, M., D. Yarlett, M. Dye, K. Denny \& K. Thorpe. 2010. The effects of feature-label-order and their implications for symbolic learning. Cognitive Science 34.6, 909-957. doi:10.1111/j.15516709.2009.01092.x.

Rescorla, R. A. 1988. Pavlovian conditioning. It's not what you think it is. American Psychologist 43.3, 151-160. doi:10.1037/0003-066X.43.3.151.

Rescorla, R. A. \& A. R. Wagner. 1972. A theory of Pavlovian conditioning: Variations in the effectiveness of reinforcement and nonreinforcement. In A. H. Black \& W. F. Prokasy (eds.), Classical conditioning II: Current research and theory, 64-99. New York: Appleton Century Crofts. 
Rose, Darcy. 2017. Predicting plurality: An examination of the effects of morphological predictability on the learning and realization of bound morphemes. Christchurch: University of Canterbury $\mathrm{PhD}$ Dissertation.

Rosenblatt, Frank. 1962. Principles of neurodynamics. Spartan Book, Washington DC.

Scarborough, R. A. 2004. Degree of coarticulation and lexical confusability. In P. M. Nowak, C. Yoquelet \& D. Mortensen (eds.), Proceedings of the 29th Meeting of the Berkeley Linguistics Society, February 14-17. Berkley: University of Berkley.

Schweitzer, A. \& N. Lewandowski. 2013. Convergence of articulation rate in spontaneous speech. Proceedings of interspeech 2013, 525-529. Lyon: International Speech Communication Association.

Seidenberg, M. 1987. Sublexical structures in visual word recognition: Access units or orthographic redundancy. In M. Coltheart (ed.), Attention and Performance XII, 245-264. Hove: Lawrence Erlbaum Associates.

Sering, K., M. Weitz, D. E. Kuenstle \& L. Schneider. 2018a. Pyndl: Nä̈ve discriminative learning in python. doi:10.5281/zenodo.1134829.

Sering, T., P. Milin \& R. H. Baayen. 2018b. Language comprehension as a multiple label classification problem. Statistica Neerlandica 72.3, 1-15. doi:10.1111/stan.12134.

Seyfarth, Scott, Marc Garellek, Gwendolyn Gillingham, Farrell Ackerman \& Robert Malouf. 2018. Acoustic differences in morphologically-distinct homophones. Language, Cognition and Neuroscience 33.1, 32-49.

Shafaei Bajestan, E. \& R. H. Baayen. 2018. Wide learning for auditory comprehension. Proceedings of interspeech 2018, 448-451. Graz, Austria: International Speech Communication Association.

Shaoul, C., N. Schilling, S. Bitschnau, A. Arppe, P. Hendrix \& R. H. Baayen. 2014. Ndl2: Nä̈ve discriminative learning. $\mathrm{R}$ package version 1.901, development version available upon request.

Shaoul, Cyrus \& C. Westbury. 2010a. Exploring lexical co-occurrence space using hidex. Behavior Research Methods 42.2, 393-413.

Shaoul, Cyrus \& Chris Westbury. 2010b. Exploring lexical co-occurrence space using HiDEx. Behavior Research Methods 42.2, 393-413. http://link.springer.com/article/10.3758/BRM.42.2. 393.

Smith, Rachel, Rachel Baker \& Sarah Hawkins. 2012. Phonetic detail that distinguishes prefixed from pseudo-prefixed words. Journal of Phonetics 40.5, 689-705. doi:10.1016/j.wocn.2012.04.002. http://www.sciencedirect.com/science/article/pii/S0095447012000356.

Song, Jae Yung, Katherine Demuth, Karen Evans \& Stefanie Shattuck-Hufnagel. 2013. Durational cues to fricative codas in 2-year-olds' American English: Voicing and morphemic factors. The Journal of the Acoustical Society of America 133.5, 2931-2946.

Tomaschek, F., D. Arnold, Franziska Bröker \& R. H. R. Baayen. 2018a. Lexical frequency codetermines the speed-curvature relation in articulation. Journal of Phonetics 68, 103-116.

Tomaschek, F., D. Arnold, J. van Rij, B. V. Tucker \& K. Sering. 2019. Proficiency effects on the movement precision during the execution of articulatory gestures (submitted manuscript).

Tomaschek, F., P. Hendrix \& R. H. Baayen. 2018b. Strategies for managing collinearity in multivariate linguistic data. Journal of Phonetics 71, 249-267.

Tomaschek, F., B. V. Tucker, R. H. Baayen \& M. Fasiolo. 2018c. Practice makes perfect: The consequences of lexical proficiency for articulation. Linguistic Vanguard 4.s2, 1-13.

Torreira, Francisco \& Mirjam Ernestus. 2009. Probabilistic effects on French [t] duration. Proceedings of interspeech 2009, 448-451. Lyon: International Speech Communication Association.

Tremblay, A., B. Derwing, G. Libben \& C. Westbury. 2011. Processing advantages of lexical bundles: Evidence from self-paced reading and sentence recall tasks. Language Learning 61.2, 569-613.

Tremblay, A. \& B. V. Tucker. 2011. The effects of n-gram probabilistic measures on the recognition and production of four-word sequences. The Mental Lexicon 6.2, 302-324.

Tucker, B. V., M. Sims \& R. H. Baayen. 2019. Opposing forces on acoustic duration. https://psyarxiv. com/jc97w, doi:10.31234/osf.io/jc97w.

Vitevitch, Michael S. 2002. The influence of phonological similarity neighborhoods on speech production. Journal of Experimental Psychology: Learning, Memory and Cognition 28.4, 735-747.

Vitevitch, Michael S. 2008. What can graph theory tell us about word learning and lexical retrieval? Journal of Speech, Language and Hearing Research 51.2, 408.

Vitevitch, Michael S. \& Melissa K. Stamer. 2006. The curious case of competition in Spanish speech production. Language and Cognitive Processes 21.6, 760-770.

Wagner, A. R. \& R. A. Rescorla. 1972. Inhibition in Pavlovian conditioning: Application of a theory. In R. A. Boakes \& M. S. Halliday (eds.), Inhibition and learning, 301-336. New York: Academic Press. 
Walsh, Liam, Jennifer Hay, Hay Jen, Bent Derek, Liz Grant, Jeanette King, Paul Millar, Viktoria Papp \& Kevin Watson. 2013. The UC QuakeBox Project: Creation of a community-focused research archive. New Zealand English Journal 27, 20-32.

Walsh, T. \& F. Parker. 1983. The duration of morphemic and non-morphemic /s/ in English. Journal of Phonetics 11, 201-206.

Wedel, A., S. Jackson \& A. Kaplan. 2013a. Functional load and the lexicon: Evidence that syntactic category and frequency relationships in minimal lemma pairs predict the loss of phoneme contrasts in language change. Language and Speech 56.3, 395-417.

Wedel, Andrew, Abby Kaplan \& Scott Jackson. 2013b. High functional load inhibits phonological contrast loss: A corpus study. Cognition 128.2, 179-186.

Weingarten, R., G. Nottbusch \& U. Will. 2004. Morphemes, syllables and graphemes in written word production. In T. Pechmann \& Ch. Habel (eds.), Multidisciplinary approaches to speech production, 529-572. Berlin: Mouton de Gruyter.

Widrow, B. \& Marcian E. Hoff et al. 1960. Adaptive switching circuits. 1960 IRE WESCON convention record, part 4, 96-104. New York: Institute of Radio Engineers.

Wood, S. N. 2006. Generalized Additive Models. New York: Chapman \& Hall/CRC.

Wood, S. N. 2011. Fast stable restricted maximum likelihood and marginal likelihood estimation of semiparametric generalized linear models. Journal of the Royal Statistical Society (B) 73, 3-36.

Wood, Simon N., Natalya Pya \& Benjamin Säfken. 2016. Smoothing parameter and model selection for general smooth models. Journal of the American Statistical Association: Theory and Methods $111.516,1548-1575$.

Zimmermann, Julia. 2016a. Morphological status and acoustic realization: Findings from New Zealand English. In Christopher Carignan \& Michael D. Tyler (eds.), Proceedings of the 16th Australasian International Conference on Speech Science and Technology, 6-9 December 2016, Parramatta, Australia. Sydney: University of Western Sydney.

Zimmermann, Julia. 2016b. Morphological status and acoustic realization: Is there a difference between Bra[d] Pitt and a grille[d] cheese omelet, or between Kate Mo[s] and killer robot[s]? In Johannes Wahle, Marisa Köllner, Harald R. Baayen, Gerhard Jäger \& Tineke Baayen-Oudshoorn (eds.), Proceedings of 6th Conference on Quantitative Investigations in Theoretical Linguistics (QITL-6). Tübingen: Universität Tübingen. http://hdl.handle.net/10900/68949.

Zipf, G. K. 1929. Relative frequency as a determinant of phonetic change. Harvard Studies in Classical Philology 15, 1-95.

Zipf, G. K. 1949. Human behavior and the principle of the least effort. An introduction to human ecology. New York: Hafner.

\author{
Authors'addresses: (Tomaschek) \\ Universität Tübingen, Wilhelmstrasse 19-23, \\ 72072 Tübingen, Germany \\ fabian.tomaschek@uni-tuebingen.de \\ (Plag) \\ Heinrich-Heine-Universität Düsseldorf, Universitätsstrasse 1, \\ 40225 Düsseldorf, Germany \\ ingo.plag@uni-duesseldorf.de \\ (Ernestus) \\ Radboud University Nijmegen, P.O. Box 9103, \\ 6500 HD Nijmegen, The Netherlands \\ m.ernestus@let.ru.nl \\ (Baayen) \\ Universität Tübingen, Wilhelmstrasse 19-23, \\ 72072 Tübingen, Germany \\ harald.baayen@uni-tuebingen.de
}

